\title{
EXTRAPOLATION OF VECTOR FIELDS USING THE INFINITY LAPLACIAN AND WITH APPLICATIONS TO IMAGE SEGMENTATION*
}

\author{
CAROLE LE GUYADER ${ }^{\dagger}$ AND LAURENCE GUILLOT ${ }^{\ddagger}$
}

\begin{abstract}
In this paper, we investigate a new Gradient-Vector-Flow (GVF)-inspired static external force field for active contour models, deriving from the edge map of a given image and allowing to increase the capture range. Contrary to prior related works, we reduce the number of unknowns to a single one $v$ by assuming that the expected vector field is the gradient field of a scalar function. The model is phrased in terms of a functional minimization problem comprising a data fidelity term and a regularizer based on the supremum norm of $D v$.

The minimization is achieved by solving a second order singular degenerate parabolic equation. A comparison principle as well as the existence/uniqueness of a viscosity solution together with regularity results are established. Experimental results for image segmentation with details of the algorithm are also presented.
\end{abstract}

Key words. Gradient Vector Flow, infinity Laplacian, AMLE, partial differential equations, viscosity solutions, segmentation.

AMS subject classifications. 35Q80, 68U10, 49L25, 35G25, 35D05, 35D10, 74G65.

\section{Introduction}

1.1. Motivations. Segmentation is the process which consists of detecting and visualizing the boundaries of the objects contained in an image. Many of the well-known variational segmentation methods require a careful choice of the initial condition. One of the most famous variational methods to process this partition of the image is the active contour model introduced by Kass, Witkin, and Terzopoulos [51]. It consists of evolving a parameterized curve so that it matches the object boundary. The shape taken by the curve through the process is related to an energy minimization, where this energy is comprised of a data fitting term and a regularizer, and is nonconvex. Therefore, we can only expect local minimizers, which, in practice, means that the contour to be deformed must be initialized near the object boundary. Cohen [30] has proposed a way to alleviate this constraint by adding an inflating/deflating force in the modelling, defined by $k \vec{n}, \vec{n}$ denoting the unit inward normal to the curve and $k$, a constant. According to the sign of the constant $k$, the curve inflates or deflates. Thereby, in practice, the contour to be deformed is either initialized inside the object, or it encloses the object of interest.

In [65], Xu and Prince address both the problems of initialization and slow/poor convergence near boundaries with strong concavities by introducing a new static external force called Gradient Vector Flow (GVF). The initialization constraint is removed, that is, initialization can be made inside, outside or across the object boundaries, and the front evolution is easily handled even in boundary concavities.

*Received: October 3, 2008, accepted (in revised version): March 14, 2009. Communicated by Luminita Vese.

${ }^{\dagger}$ IRMAR, UMR CNRS 6625, Institut National des Sciences Appliquées de Rennes, 20 Avenue des Buttes de Coësmes, CS 14315, 35043 Rennes Cedex, France and INSA Rouen, LMI, Place Emile Blondel, BP08, 76131 Mont-Saint-aignan Cedex, France (cleguyad@insa-rennes.fr).

${ }^{\ddagger}$ Laboratoire Jean-Alexandre Dieudonné, UMR 6621, Université de Nice Sophia Antipolis - CNRS, Faculté des Sciences Parc Valrose, 06108 - Nice Cedex 02, France (lguillot@unice.fr). 
The main idea behind this model is to increase the capture range of the external edge-map-related force field and to make the contour evolve toward the desired boundaries, where classical methods would fail. Unlike classical active contours, the introduced external force does not derive from a potential function and cannot be straightforwardly computed from the image edge map. More precisely, the model cannot be phrased in terms of a unique functional minimization problem but is defined in two steps. In the first step, the external force (GVF) $w=(u, v)$ is obtained by minimizing an energy functional in a variational framework. Denoting by $D f$ the gradient field of an edge map, $\mathrm{Xu}$ and Prince propose to minimize the following functional:

$$
E(w)=\mu \int_{\Omega}\left(u_{x}^{2}+u_{y}^{2}+v_{x}^{2}+v_{y}^{2}\right) d x d y+\int_{\Omega}\|D f\|^{2}\|w-D f\|^{2} d x d y
$$

with $\mu$, a tuning parameter, $\Omega$, a bounded open subset of $\mathbb{R}^{2}$, $\|\cdot\|$ denoting the euclidean norm in $\mathbb{R}^{2}$, and with the notation $u_{x}=\frac{\partial u}{\partial x}$. The energy $E$ is thus designed such that when $\|D f\|$ is large, it is minimized by setting $w=D f$, and when $\|D f\|$ is small, the resulting $w$ is smooth and slowly varying. The Euler-Lagrange equations are computed and lead to a decoupled linear partial differential equation system to be solved. Numerically, these equations are solved by a gradient descent method, and one obtains the following system:

$$
\left\{\begin{array}{l}
\frac{\partial u}{\partial t}=\mu \Delta u-2\|D f\|^{2}\left(u-f_{x}\right), \\
\frac{\partial v}{\partial t}=\mu \Delta v-2\|D f\|^{2}\left(v-f_{y}\right) .
\end{array}\right.
$$

The second step then consists in replacing, in the dynamic snake equation, the classical potential force by the newly computed external force $w$.

This method motivated the following works: in [58], Paragios et al. proposed integrating this boundary spatial diffusion technique to the geodesic active contours [28]. In [42], a proof of existence and uniqueness of the viscosity solution of this model is established using the work by Ishii and Sato [46]. In [50], Jifeng et al. proposed improving the diffusion properties of the GVF force field. They obtained a new force by replacing the Laplacian operator used in the GVF model by its diffusion term in the normal direction, that is, the 'normalized' infinity Laplacian operator (more details are given on the infinity Laplacian in the following). Numerically, the authors thus solved the following decoupled partial differential equation system:

$$
\left\{\begin{array}{l}
\frac{\partial u}{\partial t}=\mu u_{N N}-2\|D f\|^{2}\left(u-f_{x}\right), \\
\frac{\partial v}{\partial t}=\mu v_{N N}-2\|D f\|^{2}\left(v-f_{y}\right),
\end{array}\right.
$$

where $u_{N N}=\frac{u_{x}^{2} u_{x x}+2 u_{x} u_{y} u_{x y}+u_{y}^{2} u_{y y}}{\|D u\|^{2}}$.

Unlike the GVF model, their new field (called NGVF) is anisotropic. Furthermore, the NGVF is stable for larger time steps, slightly improves segmentation results, and allows faster detection of long and thin concavities.

Our work is highly motivated by $[64,65]$ and $[50]$. We wanted to provide, in a rigorous mathematical framework, a new method to generate this external force field. Contrary to these prior works, we propose to reduce the number of unknowns to a single one, by assuming that the sought vector field is the gradient field of a scalar function. Also, the minimization problem introduced by this procedure contains a 
data-fitting term related to the original GVF model and a regularizer that penalizes the supremum norm of the unknown gradient. Thus the problem becomes related to the absolutely minimizing Lipschitz extensions and the infinity Laplacian.

The absolute minimal Lipschitz extension model was introduced by Aronsson in [5] (see also $[4,6,7]$ ) in the following way. Given $\Omega \subset \mathbb{R}^{n}$ a bounded, open, and connected domain with sufficiently smooth boundary and $b \in \mathcal{C}(\partial \Omega)$, solve

$$
\inf _{u \in W^{1, \infty}(\Omega), u=b \text { on } \partial \Omega}\|D u\|_{L^{\infty}(\Omega)} .
$$

A minimizer of problem (1.1) is called an absolutely minimizing Lipschitz interpolant of $\left.b\right|_{\partial \Omega}$ inside $\Omega$. Aronsson proved the existence of an absolute minimal Lipschitz extension and Jensen proved the uniqueness. Aronsson also derived the Euler-Lagrange equation governing the absolute minimizer in the sense of viscosity solutions :

$$
\Delta_{\infty} u=D^{2} u(D u, D u)=0 \quad \text { in } \Omega .
$$

We refer to [9] and to [17] for more details. The operator $\Delta_{\infty}$ is called the infinity Laplacian and solutions of (1.2) are said to be $\infty$-harmonic (see Crandall [32] for a complete review of the infinity Laplacian equation). Jensen proved a comparison principle and an existence/uniqueness result of (1.2) for Lipschitz continuous boundary data. Also, the infinity Laplacian equation was derived as the limiting case $p \rightarrow \infty$ of the Euler-Lagrange equation $\Delta_{p} u=\operatorname{div}\left(|D u|^{p-2} D u\right)=0$ related to the homogeneous Dirichlet problem with $1<p<\infty$ :

$$
\inf _{u \in W^{1, p}(\Omega), u=b \text { on } \partial \Omega}\|D u\|_{L^{p}(\Omega)} .
$$

As stressed in [38], this limiting process $p \rightarrow \infty$ was made rigorous in $[6,49]$. Timedependent versions of equation 1.2 have been explored for instance in the image interpolation framework [29], the first one naturally derived from (1.2):

$$
\begin{aligned}
\frac{\partial u}{\partial t} & =D^{2} u(D u, D u)=u_{x_{i}} u_{x_{j}} u_{x_{i} x_{j}} \text { in } \Omega \times(0,+\infty), \\
u(x, 0) & =u_{0}(x) \text { in } \Omega, \\
u(x, t) & =b(x) \text { for }(x, t) \in \partial \Omega \times(0,+\infty),
\end{aligned}
$$

the second one being its 'normalized version':

$$
\begin{aligned}
\frac{\partial u}{\partial t} & =D^{2} u\left(\frac{D u}{|D u|}, \frac{D u}{|D u|}\right) \text { in } \Omega \times(0,+\infty), \\
u(x, 0) & =u_{0}(x) \text { in } \Omega, \\
u(x, t) & =b(x) \text { for }(x, t) \in \partial \Omega \times(0,+\infty) .
\end{aligned}
$$

The operator $D^{2} u\left(\frac{D u}{|D u|}, \frac{D u}{|D u|}\right)$ is the second derivative of $u$ in the normal direction $\frac{D u}{|D u|}$.

Before depicting our model, we make a non-exhaustive review of some prior works related to AMLE and the infinity Laplacian in the field of image processing. 


\subsection{Prior related works.}

The work of Caselles, Morel, and Sbert. As stressed by Caselles et al., the equation $\Delta_{\infty} u=D^{2} u(D u, D u)=0$ was introduced in the field of computer vision as an edge detector (see [43,61,66]). Earlier, it appeared in the domain of edge enhancement (see [59]) and served as the basis of Canny edge detection [22]. In [29], Caselles et al. investigate the AMLE and the infinity Laplacian in the field of image processing with applications to the restoration of images. Motivated by prior applications devoted to coding [24, 25, 26], they address the issue of interpolating data given on a set of points and/or curves in the plane. The set of requirements to be fulfilled by the interpolation operator (comparison, stability, regularity principles as well as independency of the interpolation process with respect to the observer's standpoint) leads to models in which the operator is given, in the case of continuous data, as the viscosity solution of a degenerate elliptic partial differential equation. The authors prove that the AMLE model is the most suitable to interpolate data given on points and/or curves, providing a result of existence/uniqueness of a Lipschitz viscosity solution.

The work of Cong, Esser, Parvin and Bebis. Another application, dedicated to shape metamorphism (the process which consists in evolving a source shape into a target shape by intermediate steps) is proposed by Cong et al. in [31] and makes use of the infinity Laplacian. Denoting $\mathcal{S}(t), t \in[0,1]$, to be a collection of deformable closed curves with known boundary conditions at $\mathcal{S}(0)$ and $\mathcal{S}(1)$, their goal is to reconstruct the intermediate curves $\mathcal{S}(t), 0<t<1$ so that the obtained sequence of curves is smooth and continuous in time. The function $\mathcal{O}_{i}$ is the inside-outside function of a closed curve $\mathcal{S}_{i}, i=0,1$,

$$
\mathcal{O}_{i}(x, y)=\left\{\begin{array}{l}
-1 \text { if }(x, y) \text { is inside } \mathcal{S}_{i} \\
1 \text { if }(x, y) \text { is outside } \mathcal{S}_{i} \\
0 \text { if }(x, y) \text { is on } \mathcal{S}_{i} .
\end{array}\right.
$$

The authors restrict the curve evolution domain to the region $\mathcal{R}$ defined by $\mathcal{R}=$ $\mathcal{R}\left(\mathcal{S}_{0}, \mathcal{S}_{1}\right)=\left\{(x, y) \mid \mathcal{O}_{0}(x, y) \mathcal{O}_{1}(x, y) \leq 0\right\}$. Then they use an implicit representation of the intermediate curves, expressed as the level curves of a function $f$ :

$$
\mathcal{S}(t)=\{(x, y) \mid f(x, y)=t\}
$$

which leads to the following problem:

$$
\text { 'Find } f(x, y),(x, y) \in \mathcal{R} \text {, such that } f\left(\mathcal{S}_{0}\right)=0, f\left(\mathcal{S}_{1}\right)=1 .
$$

This underconstrained problem is complemented by a regularization on $f$ which is the supremum norm of $D f$, with the aim to control $f$ locally, and is stated as:

'Find $f(x, y),(x, y) \in \mathcal{R}$, such that $f\left(\mathcal{S}_{0}\right)=0, f\left(\mathcal{S}_{1}\right)=1$ and $\Delta_{\infty} f=0$ ''

The authors prove that their model is optimal for metamorphism since the intermediate solutions are equally distributed along their normal directions.

The work of Mémoli, Sapiro and Thompson. In [54], Mémoli et al. propose a new framework for brain warping using Minimizing Lipschitz Extensions. Let $\mathcal{B}_{1}$ and $\mathcal{B}_{2}$ be the two cortical surfaces to be matched, and let $\Gamma_{1}=\cup_{i=1}^{N} x_{i} \subset \mathcal{B}_{1}$, $\Gamma_{2}=\cup_{i=1}^{N} y_{i} \subset \mathcal{B}_{2}$ be two sets of landmark points for which a correspondence is already known. The authors aim at finding a sufficiently smooth mapping $\Phi: \mathcal{B}_{1} \mapsto \mathcal{B}_{2}$ such 
that $\Phi\left(x_{i}\right)=y_{i}$ for $1 \leq i \leq N$ and such that $\Phi$ produces minimal distortion according to a given functional $J$.

This extrapolation problem is solved by minimizing $J_{\infty}(\Phi)=$ $\operatorname{essup}_{x \in \mathcal{B}_{1}}\left\|D_{\mathcal{B}_{1}} \Phi(x)\right\|$ over the space of all Lipschitz continuous mappings $\Psi: \mathcal{B}_{1} \mapsto \mathcal{B}_{2}$ such that $\Psi\left(x_{i}\right)=y_{i}$ for $1 \leq i \leq N$.

The work of Elion and Vese. In [55], Meyer introduced the following image decomposition model in which the image $f$ is broken down into a geometrical part $u$ and an oscillatory or texture part $v$ :

$$
\inf _{\substack{f=u+v \\(u, v) \in(B V \times G)}} E(u)=\int|D u|+\lambda\|v\|_{G},
$$

where the space $G$ containing $v$ is the Banach space of distributions $v=\operatorname{div} \vec{g}, g_{1}, g_{2} \in$ $L^{\infty}\left(\mathbb{R}^{2}\right)$ endowed with the norm

$$
\|v\|_{G\left(\mathbb{R}^{2}\right)}=\inf _{\substack{\vec{g}=\left(g_{1}, g_{2}\right) \in\left(L^{\infty}\left(\mathbb{R}^{2}\right)\right)^{2} \\ v=\operatorname{div} \vec{g}}}\|\vec{g}\|_{L^{\infty}\left(\mathbb{R}^{2}\right)}
$$

The space $G$ allows oscillating functions $v$, and the oscillations are measured by the norm $\|v\|_{G}$. However, due to the definition of the $G$-norm the Euler-Lagrange equations associated with the minimization problem (1.4) cannot be written. Different approximations have been proposed by Vese and Osher in [62, 63], by Aujol et al. in $[12,13,11]$, and Le and Vese in [52]. In [38], Elion and Vese aim at solving the $(B V, G)$ decomposition model. In that purpose, they focus on an isotropic decomposition of the image $f \approx u+v$ with $v=\Delta P=\operatorname{div}(D P)$ and $D P \in\left(L^{\infty}(\Omega)\right)^{2}$. Given $f \in L^{2}(\Omega)$, their proposed model is

$$
\inf _{\substack{u \in B V(\Omega) \\ D P \in\left(L^{\infty}(\Omega)\right)^{2}, \Delta P \in L^{2}(\Omega)}} F(u, P)=\int|D u| d x+\mu \int|f-(u+\Delta P)|^{2} d x+\lambda\|D P\|_{L^{\infty}(\Omega)} .
$$

The outline of the paper is as follows. Section 2 is devoted to the depiction of the model and the derivation of the associated evolution problem. Section 3 is dedicated to the theoretical study of the obtained parabolic problem. We first prove a comparison principle, then prove existence and uniqueness of a viscosity solution. Regularity results of this solution are also given. We conclude the paper with experimental results and integrate this new external force field in a segmentation problem. Details of the algorithm are also provided.

\section{Depiction of the model}

Let $\Omega$ be a bounded open subset of $\mathbb{R}^{n}$, its boundary denoted by $\partial \Omega$, and let $I$ be a given bounded image function defined by $I: \bar{\Omega} \rightarrow \mathbb{R}$. For the purpose of illustration we consider $n=2$.

Let $g$ be an edge-detector map. The function $g$ is applied to the norm of the image gradient, and satisfies the following properties: $g:[0, \infty[\rightarrow[0, \infty[, g(0)=1, g$ strictly decreasing, and $\lim _{r \rightarrow+\infty} g(r)=0$. An example of such a function is $g: r \mapsto \frac{1}{1+r^{2}}$. We denote $W=\left(w_{1}, w_{2}\right)=-D g(\|D I\|)$ to be the associated gradient vector field. In homogeneous regions, $\|D I\| \simeq 0$ so $g(\|D I\|)$ is almost equal to 1 . On boundaries, $\|D I\|$ is large so $g(\|D I\|)$ is almost zero. Also, in homogeneous regions $W$ is almost 
the null vector. Along the boundaries, the vector field $W$ points toward the middle of the edges (see such an example in figure 4.1).

We plan to extrapolate the vector field on the whole image domain in a variational framework.

For completeness, we also refer the reader to $[2,18,19,20,36,37,53]$ which address the issue of vector field approximation, even if they are not directly related to the main ingredients of the proposed approach, and to [3] for a general introduction to the $D^{m}$-spline theory that is used for instance in [53].

A majority of existing regularization functionals aims at minimizing the global variation of the unknowns and thus provides little local control. In this work, we propose to minimize the supremum norm of the unknown gradient. Also, unlike prior related works, we reduce the number of unknowns to a single one by assuming that the expected vector field is the gradient vector field of a scalar function. We thus propose to minimize the following functional:

$$
\inf _{v \in W^{1, \infty}(\Omega)} \int_{\Omega}\|D v-W\|^{2}\|W\|^{2} d x+\mu\|D v\|_{L^{\infty}(\Omega)},
$$

where $\mu>0$ is a tuning parameter.

Remark 2.1. Functional (2.1) is defined on $W^{1, \infty}(\Omega)$. The domain $\Omega$ is bounded, and the inclusion $L^{\infty}(\Omega) \subset L^{2}(\Omega)$ holds so $D v \in L^{2}(\Omega)$.

Remark 2.2. If $v$ is a minimizer of (2.1), then so is $v+C$ where $C$ denotes any real constant. This is not a problem since we are interested in the associated gradient vector field. If $v \in W^{1, \infty}(\Omega), v$ is Lipschitz continuous and thus, by Rademacher's theorem, differentiable almost everywhere.

To minimize the above energy, we make use of the absolutely minimizing Lipschitz extensions. Following the results on AMLE recalled in section 1, we obtain the EulerLagrange equation satisfied by $v$ if it minimizes (2.1) and solve it by gradient descent. More precisely, classically, in image processing, the equation is defined on a domain $R$ of $\mathbb{R}^{2}$ (e.g. on the square $\left.[0,1] \times[0,1]\right)$. In this case, boundary conditions must be defined: Neumann boundary conditions on $\partial R$ are well-suited to the image processing framework since it corresponds to the reflection of the data through the edges. Thus it is no longer necessary to define boundary values.

Following [10] and [27], we propose to simplify the problem by working with periodic solutions. The function $v$, primarily defined on $[0,1] \times[0,1]$, is extended to $\mathbb{R}^{2}$. First, by symmetry, we extend it to $[-1,1] \times[-1,1]$ and then in all of $\mathbb{R}^{2}$ by periodicity (see section 3.3.1 from [10]). We thus obtain that $\forall h \in \mathbb{Z}^{2}, \forall x \in \mathbb{R}^{2}, v(x+2 h)=v(x)$. Also, we assume that the initial condition $v_{0}$ and the functions $x \mapsto w_{k}(x), k=1,2$ are extended to $\mathbb{R}^{2}$ with the same periodicity.

Given $T>0$, we then obtain the following problem:

$$
\left\{\begin{aligned}
& \frac{\partial v}{\partial t}= 2\|W\|^{2} \Delta v+2\left\langle D\|W\|^{2}, D v\right\rangle-2 \operatorname{div}\left(\|W\|^{2} W\right)+\mu D^{2} v\left(\frac{D v}{|D v|}, \frac{D v}{|D v|}\right) \\
& \text { on } \mathbb{R}^{2} \times(0, T), \\
&= b(x) \Delta v-\langle d(x), D v\rangle-h(x)+\mu D^{2} v\left(\frac{D v}{|D v|}, \frac{D v}{|D v|}\right), \\
& v(x, t=0)=v_{0}(x) \text { in } \mathbb{R}^{2},
\end{aligned}\right.
$$

with $b: x \mapsto 2\|W(x)\|^{2}, d: x \mapsto-2 D\|W\|^{2}(x), h: x \mapsto 2 \operatorname{div}\left(\|W\|^{2} W\right)(x)$, and with the assumptions $v_{0} \in \mathcal{C}\left(\mathbb{R}^{2}\right) \cap W^{1, \infty}\left(\mathbb{R}^{2}\right), b \in \mathcal{C}\left(\mathbb{R}^{2}\right)$ and bounded by $\xi_{b}, d \in \mathcal{C}\left(\mathbb{R}^{2}\right) \cap$ 
$W^{1, \infty}\left(\mathbb{R}^{2}\right)$, bounded by $\xi_{d}$ and with Lipschitz constant $\kappa_{d}, h \in \mathcal{C}\left(\mathbb{R}^{2}\right) \cap W^{1, \infty}\left(\mathbb{R}^{2}\right)$, bounded by $\xi_{h}$ and with Lipschitz constant $\kappa_{h}$, and with $\langle\cdot, \cdot\rangle$ denoting the euclidean scalar product in $\mathbb{R}^{2}$. We also assume that the mapping $\mathbb{R}^{2} \ni x \mapsto b^{\frac{1}{2}}(x)$ is Lipschitz continuous on $\mathbb{R}^{2}$ with Lipschitz constant $\kappa_{b^{\frac{1}{2}}}$.

\section{Theoretical results}

This problem falls within the framework of the theory of viscosity solutions. Indeed, we obtain a second order singular degenerate parabolic equation.

The concept of viscosity solutions was introduced in 1981 by Crandall and Lions [34]. This theory was developed to study first-order partial differential equations of nondivergence form, typically, Hamilton-Jacobi equations. Later, the study of viscosity solutions was extended to second-order elliptic and parabolic equations (for a good introduction to the theory of viscosity solutions, we refer to Barles [15, 14], the article of Crandall, Ishii and Lions [33], Crandall, Lions [35], Ishii [44], and Ishii, Lions [45]). We also refer to the related work [16].

In our problem, the evolution equation in (2.2) can be rewritten in the form:

$$
\frac{\partial v}{\partial t}+G\left(x, D v, D^{2} v\right)=0,
$$

with $G: \mathbb{R}^{2} \times \mathbb{R}^{2}-\left\{0_{\mathbb{R}^{2}}\right\} \times \mathcal{S}^{2}\left(\mathcal{S}^{2}\right.$ being the set of symmetric $2 \times 2$ matrices equipped with its natural partial order) defined by

$$
\begin{aligned}
G(x, p, X) & =\langle d(x), p\rangle+h(x)-b(x) \operatorname{trace}(X)-\mu \frac{p^{T}}{|p|} X \frac{p}{|p|}, \\
& =\langle d(x), p\rangle+h(x)-b(x) \operatorname{trace}(X)-\mu \operatorname{trace}\left(\frac{p \otimes p}{|p|^{2}} X\right), \\
& =c(x, p)+E(x, X)+F(p, X),
\end{aligned}
$$

with the following properties.

1. The operators $G, \quad E:(x, X) \mapsto-b(x) \operatorname{trace}(X) \quad$ and $\quad F:(p, X) \mapsto$ $-\mu$ trace $\left(\frac{p \otimes p}{|p|^{2}} X\right)$ are independent of $v$ and are elliptic, i.e., $\forall X, Y \in \mathcal{S}^{2}$, $\forall p \in \mathbb{R}^{2}$,

$$
\text { if } X \leq Y \text { then } F(p, X) \geq F(p, Y) \text {. }
$$

The operators $G, E$, and $F$ are therefore proper.

2. $F$ is locally bounded on $\mathbb{R}^{2} \times \mathcal{S}^{2}$, continuous on $\mathbb{R}^{2} \backslash\left\{0_{\mathbb{R}^{2}}\right\} \times \mathcal{S}^{2}$, and

$$
F^{*}(0,0)=F_{*}(0,0)=0,
$$

where $F^{*}$ (resp. $F_{*}$ ) is the upper semicontinuous (usc) envelope (resp. lower semicontinuous (lsc) envelope) of $F$.

3. $c: \mathbb{R}^{2} \times \mathbb{R}^{2} \ni(x, p) \mapsto\langle d(x), p\rangle+h(x)$ is locally Lipschitz continuous in space and $\forall x, y \in \mathbb{R}^{2} \times \mathbb{R}^{2}$,

$$
|c(x, p)-c(y, p)| \leq\left(\kappa_{d}|p|+\kappa_{h}\right)|x-y| .
$$

For the sake of clarity, we recall some definitions that will be used in the following. 
DeFinition 3.1. We define the following sets:

$U S C\left(\mathbb{R}^{n} \times[0, T)\right)=\left\{u: \mathbb{R}^{n} \times[0, T) \rightarrow \mathbb{R}\right.$ locally bounded, upper semicontinuous $\}$.

$L S C\left(\mathbb{R}^{n} \times[0, T)\right)=\left\{u: \mathbb{R}^{n} \times[0, T) \rightarrow \mathbb{R}\right.$ locally bounded, lower semicontinuous $\}$.

Definition 3.2 (Parabolic sub and superdifferentials of semicontinuous functions.). If $u: \mathbb{R}^{n} \times(0, T) \rightarrow \mathbb{R}$, then $\mathcal{P}^{+} u$ is defined as follows:

$(a, p, X) \in \mathbb{R} \times \mathbb{R}^{n} \times \mathcal{S}^{n}$ belongs to $\mathcal{P}^{+} u(x, t)$ if $(x, t) \in \mathbb{R}^{n} \times(0, T)$ and

$$
u(y, s) \leq u(x, t)+a(s-t)+\langle p, y-x\rangle+\frac{1}{2}\langle X(y-x), y-x\rangle+o\left(|s-t|+|y-x|^{2}\right),
$$

as $\mathbb{R}^{n} \times(0, T) \ni(y, s) \rightarrow(x, t)$. Similarly, $\mathcal{P}^{-} u=-\mathcal{P}^{+}(-u)$. We also define the following two sets:

$$
\overline{\mathcal{P}}^{+} u(x, t)=\left\{\begin{array}{l}
(a, p, X) \in \mathbb{R} \times \mathbb{R}^{n} \times \mathcal{S}^{n} \\
\exists\left(x_{n}, t_{n}, a_{n}, p_{n}, X_{n}\right) \in \mathbb{R}^{n} \times \mathbb{R} \times \mathbb{R} \times \mathbb{R}^{n} \times \mathcal{S}^{n} \\
\text { such that }\left(a_{n}, p_{n}, X_{n}\right) \in \mathcal{P}^{+} u\left(x_{n}, t_{n}\right) \\
\text { and }\left(x_{n}, t_{n}, u\left(x_{n}, t_{n}\right), a_{n}, p_{n}, X_{n}\right) \rightarrow(x, t, u(x, t), a, p, X)
\end{array}\right\} .
$$

We start by proving a comparison principle that will be useful to prove the uniqueness of the viscosity solution of the considered problem.

THEOREM 3.1 (Comparison principle). Let $u \in U S C\left(\mathbb{R}^{2} \times[0, T)\right.$ ), be bounded, periodic (with the same periodicity as the initial condition of problem (2.2)), and a subsolution and $v \in L S C\left(\mathbb{R}^{2} \times[0, T)\right.$ ), be bounded, periodic (with the same periodicity as the initial condition of problem (2.2)), and a supersolution of (2.2). Assume that $u_{0}(x)=u(x, 0) \leq v_{0}(x)=v(x, 0)$ in $\mathbb{R}^{2}$. Then $u \leq v$ in $\mathbb{R}^{2} \times[0, T)$.

Proof. This proof is rather classical. For the reader's convenience, the main steps of this proof can be found in Appendix A.

We now give an existence result using the classic Perron's method (see section 4 from [33]).

We start by constructing a subsolution $U^{-}$. Let us set $U^{-}=\inf _{\mathbb{R}^{2}}\left(v_{0}\right)-C t$ with $C=\xi_{h} . U^{-}$is twice differentiable in space, once differentiable in time, bounded, periodic with the same periodicity as $v_{0}$ and $U^{-}$is a subsolution of (2.2).

Similarly, $U^{+}=\sup _{\mathbb{R}^{2}}\left(v_{0}\right)+C t$ is a supersolution of $(2.2)$. Obviously, $U^{-}(x, 0) \leq$ $U^{+}(x, 0)$. We can define:

$$
v=\sup \left\{w ; w \text { periodic with the same periodicity as } v_{0} \text {, subsolution such that } U^{-} \leq w \leq U^{+}\right\} .
$$

In that case, Perron's method states that $v$ is a periodic discontinuous solution of (2.2) with the same periodicity as $v_{0}$. Clearly, the solution is bounded since $U^{+}$is bounded. Also as $v$ is a solution, $v^{*}$ is a subsolution and $v_{*}$ a supersolution so from the comparison principle $v^{*} \leq v_{*}$. But $v_{*} \leq v^{*}$ so $v^{*}=v_{*}=v$, which gives that $v$ is continuous on $\mathbb{R}^{2} \times[0, T)$.

CONCLUSION 3.1. We have proved the existence and uniqueness of a bounded, periodic, continuous on $\mathbb{R}^{2} \times[0, T)$ viscosity solution of problem (2.2).

We now prove that a solution of problem (2.2) is Lipschitz continuous in space, and uniformly continuous in time. 
THEOREM 3.2 (Regularity results). Let us assume that $\left\|D v_{0}\right\|_{L^{\infty}\left(\mathbb{R}^{2}\right)} \leq B_{0}$ with $B_{0}>0$. Then the solution of (2.2) satisfies

$$
\|D v(\cdot, t)\|_{L^{\infty}\left(\mathbb{R}^{2}\right)} \leq B(t),
$$

with $B(t)=\kappa_{h} \frac{e^{\alpha t}-1}{\alpha}+B_{0} e^{\alpha t}$, and with $\alpha=8 \kappa_{b^{\frac{1}{2}}}^{2}+\kappa_{d}$.

Proof. The function $v$ is bounded, continuous on $\mathbb{R}^{2} \times[0, T)$, and periodic with the same periodicity as $v_{0}$. We set $\Phi^{\epsilon}(x, y, t)=B(t)\left(|x-y|^{2}+\epsilon^{2}\right)^{\frac{1}{2}}$ and aim at proving that $v(x, t)-v(y, t) \leq \Phi^{\epsilon}(x, y, t)$.

Let us set $M=\sup _{(x, y) \in \mathbb{R}^{2} \times \mathbb{R}^{2}, t \in[0, T)}\left(v(x, t)-v(y, t)-\Phi^{\epsilon}(x, y, t)\right)$. Let us assume that $M>0$. Then we denote $\bar{M}$ to be

$$
\bar{M}=\sup _{(x, y) \in \mathbb{R}^{2} \times \mathbb{R}^{2}, t \in[0, T)}\left(v(x, t)-v(y, t)-\Phi^{\epsilon}(x, y, t)-\frac{\gamma}{T-t}\right) .
$$

For $\gamma$ small enough, we have $\bar{M}>0$. Let $(\bar{x}, \bar{y}, \bar{t})$ be a maximum point with obviously $\bar{x} \neq \bar{y}$.

Let us prove that $\bar{t}>0$. Assuming the contrary, we have

$$
\begin{aligned}
& v_{0}(\bar{x})-v_{0}(\bar{y})-\Phi^{\epsilon}(\bar{x}, \bar{y}, 0)>0, \\
& v_{0}(\bar{x})-v_{0}(\bar{y})>B_{0}\left(|\bar{x}-\bar{y}|^{2}+\epsilon^{2}\right)^{\frac{1}{2}}>B_{0}|\bar{x}-\bar{y}|,
\end{aligned}
$$

which contradicts the assumptions on $v_{0}$.

Mere calculus gives

$$
\begin{gathered}
D_{x} \Phi^{\epsilon}(\bar{x}, \bar{y}, \bar{t})=B(\bar{t}) \frac{(\bar{x}-\bar{y})}{\left(|\bar{x}-\bar{y}|^{2}+\epsilon^{2}\right)^{\frac{1}{2}}} \neq 0, D_{y} \Phi^{\epsilon}(\bar{x}, \bar{y}, \bar{t})=-B(\bar{t}) \frac{(\bar{x}-\bar{y})}{\left(|\bar{x}-\bar{y}|^{2}+\epsilon^{2}\right)^{\frac{1}{2}}} \neq 0, \\
\partial_{t} \Phi^{\epsilon}(\bar{x}, \bar{y}, \bar{t})=\tau=B^{\prime}(\bar{t})\left(|\bar{x}-\bar{y}|^{2}+\epsilon^{2}\right)^{\frac{1}{2}}=e^{\alpha \bar{t}}\left(\kappa_{h}+\alpha B_{0}\right)\left(|\bar{x}-\bar{y}|^{2}+\epsilon^{2}\right)^{\frac{1}{2}},
\end{gathered}
$$

and

$$
A=D^{2} \Phi^{\epsilon}(\bar{x}, \bar{y}, \bar{t})=B(\bar{t})\left(\begin{array}{cc}
U & -U \\
-U & U
\end{array}\right)
$$

with $U=\left(|\bar{x}-\bar{y}|^{2}+\epsilon^{2}\right)^{-\frac{1}{2}} I-\left(|\bar{x}-\bar{y}|^{2}+\epsilon^{2}\right)^{-\frac{3}{2}}(\bar{x}-\bar{y}) \otimes(\bar{x}-\bar{y})$.

Using Theorem 8.3 from [33] with $\Phi(x, y, t)=\Phi^{\epsilon}(x, y, t)+\frac{\gamma}{T-t}$, for all $\eta>0$ there exist $\tau_{1}, \tau_{2} \in \mathbb{R}$ and $X, Y \in \mathcal{S}(2)$ such that

$$
\begin{aligned}
& \tau=\tau_{1}-\tau_{2}=B^{\prime}(\bar{t})\left(|\bar{x}-\bar{y}|^{2}+\epsilon^{2}\right)^{\frac{1}{2}}+\frac{\gamma}{(T-\bar{t})^{2}} \\
& \left(\tau_{1}, p_{1}, X\right) \in \overline{\mathcal{P}}^{+} v(\bar{x}, \bar{t}), \quad\left(\tau_{2}, p_{2}, Y\right) \in \overline{\mathcal{P}}^{-} v(\bar{y}, \bar{t}), \\
& -\left(\frac{1}{\eta}+\|A\|\right) I \leq\left(\begin{array}{cc}
X & 0 \\
0 & -Y
\end{array}\right) \leq A+\eta A^{2}
\end{aligned}
$$


with $p_{1}=D_{x} \Phi^{\epsilon}(\bar{x}, \bar{y}, \bar{t})$ and $p_{2}=p_{1}$.

We choose $\eta=\frac{1}{B(\bar{t})}\left(|\bar{x}-\bar{y}|^{2}+\epsilon^{2}\right)^{1 / 2}>0$, so $A+\eta A^{2}$ can be written as

with

$$
A+\eta A^{2}=B(\bar{t})\left(\begin{array}{cc}
C & -C \\
-C & C
\end{array}\right)
$$

$$
\begin{aligned}
C= & 3\left(|\bar{x}-\bar{y}|^{2}+\epsilon^{2}\right)^{\frac{-1}{2}} I-5\left(|\bar{x}-\bar{y}|^{2}+\epsilon^{2}\right)^{\frac{-3}{2}}(\bar{x}-\bar{y}) \bigotimes(\bar{x}-\bar{y}) \\
& +2|\bar{x}-\bar{y}|^{2}\left(|\bar{x}-\bar{y}|^{2}+\epsilon^{2}\right)^{\frac{-5}{2}}(\bar{x}-\bar{y}) \bigotimes(\bar{x}-\bar{y}) .
\end{aligned}
$$

So the following holds

$$
\begin{gathered}
\tau_{1}+c\left(\bar{x}, \frac{B(\bar{t})(\bar{x}-\bar{y})}{\left(|\bar{x}-\bar{y}|^{2}+\epsilon^{2}\right)^{\frac{1}{2}}}\right)+E(\bar{x}, X)+F\left(\frac{B(\bar{t})(\bar{x}-\bar{y})}{\left(|\bar{x}-\bar{y}|^{2}+\epsilon^{2}\right)^{\frac{1}{2}}}, X\right) \leq 0, \\
\tau_{2}+c\left(\bar{y}, \frac{B(\bar{t})(\bar{x}-\bar{y})}{\left(|\bar{x}-\bar{y}|^{2}+\epsilon^{2}\right)^{\frac{1}{2}}}\right)+E(\bar{y}, Y)+F\left(\frac{B(\bar{t})(\bar{x}-\bar{y})}{\left(|\bar{x}-\bar{y}|^{2}+\epsilon^{2}\right)^{\frac{1}{2}}}, Y\right) \geq 0 .
\end{gathered}
$$

From the matrix inequality, $X \leq Y$ and using the ellipticity of $F$, we have

$$
F\left(\frac{B(\bar{t})(\bar{x}-\bar{y})}{\left(|\bar{x}-\bar{y}|^{2}+\epsilon^{2}\right)^{\frac{1}{2}}}, X\right)-F\left(\frac{B(\bar{t})(\bar{x}-\bar{y})}{\left(|\bar{x}-\bar{y}|^{2}+\epsilon^{2}\right)^{\frac{1}{2}}}, Y\right) \geq 0
$$

and

$$
c\left(\bar{x}, \frac{B(\bar{t})(\bar{x}-\bar{y})}{\left(|\bar{x}-\bar{y}|^{2}+\epsilon^{2}\right)^{\frac{1}{2}}}\right)-c\left(\bar{y}, \frac{B(\bar{t})(\bar{x}-\bar{y})}{\left(|\bar{x}-\bar{y}|^{2}+\epsilon^{2}\right)^{\frac{1}{2}}}\right) \geq-\left(\kappa_{d} B(\bar{t})+\kappa_{h}\right)|\bar{x}-\bar{y}| .
$$

We finally obtain by substracting the two relations from (3.4)

$$
\tau_{1}-\tau_{2}+b(\bar{y}) \operatorname{trace}(Y)-b(\bar{x}) \operatorname{trace}(X)-\left(\kappa_{d} B(\bar{t})+\kappa_{h}\right)|\bar{x}-\bar{y}| \leq 0,
$$

that is,

$$
\begin{aligned}
& B^{\prime}(\bar{t})\left(|\bar{x}-\bar{y}|^{2}+\epsilon^{2}\right)^{\frac{1}{2}}+\frac{\gamma}{(T-\bar{t})^{2}}+b(\bar{y}) \operatorname{trace}(Y)-b(\bar{x}) \operatorname{trace}(X) \\
& -\left(\kappa_{d} B(\bar{t})+\kappa_{h}\right)|\bar{x}-\bar{y}| \leq 0 .
\end{aligned}
$$

But,

$$
\begin{aligned}
b(\bar{x}) \operatorname{trace}(X)-b(\bar{y}) \operatorname{trace}(Y) & =\operatorname{trace}\left(\left(\begin{array}{cc}
b(\bar{x}) & \sqrt{b(\bar{x}) b(\bar{y})} \\
\sqrt{b(\bar{x}) b(\bar{y})} & b(\bar{y})
\end{array}\right)\left(\begin{array}{cc}
X & 0 \\
0 & -Y
\end{array}\right)\right), \\
& \leq B(\bar{t})(\sqrt{b(\bar{x})}-\sqrt{b(\bar{y})})^{2} \operatorname{trace}(C), \\
& \leq B(\bar{t}) \kappa_{b^{\frac{1}{2}}}^{2}|\bar{x}-\bar{y}|^{2} \operatorname{trace}(C),
\end{aligned}
$$

with

$$
\operatorname{trace}(C)=6\left(|\bar{x}-\bar{y}|^{2}+\epsilon^{2}\right)^{\frac{-1}{2}}-5|\bar{x}-\bar{y}|^{2}\left(|\bar{x}-\bar{y}|^{2}+\epsilon^{2}\right)^{\frac{-3}{2}}+2|\bar{x}-\bar{y}|^{4}\left(|\bar{x}-\bar{y}|^{2}+\epsilon^{2}\right)^{\frac{-5}{2}} .
$$


So it yields

$$
\begin{gathered}
b(\bar{x}) \operatorname{trace}(X)-b(\bar{y}) \operatorname{trace}(Y) \\
\leq B(\bar{t}) \kappa_{b^{\frac{1}{2}}}^{2}\left(6|\bar{x}-\bar{y}|^{2}\left(|\bar{x}-\bar{y}|^{2}+\epsilon^{2}\right)^{\frac{-1}{2}}-5|\bar{x}-\bar{y}|^{4}\left(|\bar{x}-\bar{y}|^{2}+\epsilon^{2}\right)^{\frac{-3}{2}}\right. \\
\left.+2|\bar{x}-\bar{y}|^{6}\left(|\bar{x}-\bar{y}|^{2}+\epsilon^{2}\right)^{\frac{-5}{2}}\right)
\end{gathered}
$$

and

$$
\begin{aligned}
& b(\bar{y}) \operatorname{trace}(Y)-b(\bar{x}) \operatorname{trace}(X) \\
\geq & -6 B(\bar{t}) \kappa_{b^{\frac{1}{2}}}^{2}|\bar{x}-\bar{y}|^{2}\left(|\bar{x}-\bar{y}|^{2}+\epsilon^{2}\right)^{\frac{-1}{2}}-2 B(\bar{t}) \kappa_{b^{\frac{1}{2}}}^{2}|\bar{x}-\bar{y}|^{6}\left(|\bar{x}-\bar{y}|^{2}+\epsilon^{2}\right)^{\frac{-5}{2}} .
\end{aligned}
$$

So

$$
\begin{gathered}
B^{\prime}(\bar{t})\left(|\bar{x}-\bar{y}|^{2}+\epsilon^{2}\right)^{\frac{1}{2}}+\frac{\gamma}{(T-\bar{t})^{2}}-6 B(\bar{t}) \kappa_{b^{\frac{1}{2}}}^{2}|\bar{x}-\bar{y}|^{2}\left(|\bar{x}-\bar{y}|^{2}+\epsilon^{2}\right)^{\frac{-1}{2}} \\
-2 B(\bar{t}) \kappa_{b^{\frac{1}{2}}}^{2}|\bar{x}-\bar{y}|^{6}\left(|\bar{x}-\bar{y}|^{2}+\epsilon^{2}\right)^{\frac{-5}{2}}-\left(\kappa_{d} B(\bar{t})+\kappa_{h}\right)|\bar{x}-\bar{y}| \leq 0 .
\end{gathered}
$$

But,

$$
\begin{gathered}
\left(|\bar{x}-\bar{y}|^{2}+\epsilon^{2}\right)^{\frac{1}{2}}\left(B^{\prime}(\bar{t})-6 B(\bar{t}) \kappa_{b^{\frac{1}{2}}}^{2} \frac{|\bar{x}-\bar{y}|^{2}}{|\bar{x}-\bar{y}|^{2}+\epsilon^{2}}-2 B(\bar{t}) \kappa_{b^{\frac{1}{2}}}^{2} \frac{|\bar{x}-\bar{y}|^{6}}{\left(|\bar{x}-\bar{y}|^{2}+\epsilon^{2}\right)^{3}}\right. \\
\left.-\left(\kappa_{d} B(\bar{t})+\kappa_{h}\right) \frac{|\bar{x}-\bar{y}|}{\left(|\bar{x}-\bar{y}|^{2}+\epsilon^{2}\right)^{\frac{1}{2}}}\right) \\
\geq\left(|\bar{x}-\bar{y}|^{2}+\epsilon^{2}\right)^{\frac{1}{2}}\left(B^{\prime}(\bar{t})-8 \kappa_{b^{\frac{1}{2}}}^{2} B(\bar{t})-\kappa_{d} B(\bar{t})-\kappa_{h}\right) \geq 0 .
\end{gathered}
$$

Consequently, $\frac{\gamma}{(T-\bar{t})^{2}} \leq 0$ which is absurd. So $v(x, t)-v(y, t) \leq \Phi^{\epsilon}(x, y, t)$ and letting $\epsilon$ tend to 0 , one obtains

$$
v(x, t)-v(y, t) \leq B(t)|x-y|
$$

Exchanging $x$ and $y$ yields

$$
|v(x, t)-v(y, t)| \leq B(t)|x-y|
$$

TheOREM 3.3 (Regularity results). The solution $v$ is uniformly continuous in time.

Proof. We proceed as in [40]. In a first step, we assume that $v_{0}$ is bounded, periodic, $\mathcal{C}^{2}$, and such that there exists $C,\left\|D v_{0}\right\|_{L^{\infty}\left(\mathbb{R}^{2}\right)},\left\|D^{2} v_{0}\right\|_{L^{\infty}\left(\mathbb{R}^{2}\right)} \leq C$. Let us set $C_{1}=\sup _{x \in \mathbb{R}^{2}}\left(\zeta+E\left(x, D^{2} v_{0}\right)+F_{*}\left(D v_{0}, D^{2} v_{0}\right), \zeta-E\left(x, D^{2} v_{0}\right)-F^{*}\left(D v_{0}, D^{2} v_{0}\right)\right)$ with $\zeta=\xi_{d}\left\|D v_{0}\right\|_{L^{\infty}\left(\mathbb{R}^{2}\right)}+\xi_{h}$. Let us also set $v^{-}=v_{0}-C_{1} t$ and $v^{+}=v_{0}+C_{1} t$. It can be checked that $v^{-}$is a subsolution of $(2.2)$ and $v^{+}$is a supersolution. 
Using classical arguments and the comparison principle, we obtain after intermediate steps that

$$
|v(x, t+h)-v(x, t)| \leq C_{1} h
$$

We now assume that $v_{0}$ is only bounded, periodic and Lipschitz continuous, and use mollification. Let us define $\rho$ to be a standard mollifier (see chapter IV from [21] and section 2.5 from $[10]): \rho \in \mathcal{C}_{c}^{\infty}\left(\mathbb{R}^{2}\right), \rho \geq 0, \operatorname{supp}(\rho) \subset B(0,1)$, and $\int_{\mathbb{R}^{2}} \rho d x=1$. For each $\epsilon>0$, let us set: $\rho_{\epsilon}(\cdot)=\frac{1}{\epsilon^{2}} \rho\left(\frac{\dot{ }}{\epsilon}\right)$. The functions $\rho_{\epsilon}$ are $\mathcal{C}_{c}^{\infty}\left(\mathbb{R}^{2}\right)$ and $\int_{\mathbb{R}^{2}} \rho_{\epsilon} d x=1$, and $\operatorname{supp}\left(\rho_{\epsilon}\right) \subset B(0, \epsilon)$. We set $v_{\epsilon}^{0}=v_{0} * \rho_{\epsilon}$. Obviously, $v_{\epsilon}^{0}$ has the same periodicity as $v_{0}$.

$$
\left|v_{\epsilon}^{0}(x)\right|=\left|v_{0} * \rho_{\epsilon}(x)\right|=\left|\int_{\mathbb{R}^{2}} v_{0}(x-y) \rho_{\epsilon}(y) d y\right| \leq\left\|v_{0}\right\|_{L^{\infty}\left(\mathbb{R}^{2}\right)} \int_{\mathbb{R}^{2}} \rho_{\epsilon}(y) d y=\left\|v_{0}\right\|_{L^{\infty}\left(\mathbb{R}^{2}\right)} .
$$

Consequently, $v_{\epsilon}^{0}$ is bounded. By the same way, we prove that $v_{\epsilon}^{0}$ is $\mathcal{C}^{2}$ and such that there exists $C$ such that $\left\|D v_{\epsilon}^{0}\right\|_{L^{\infty}\left(\mathbb{R}^{2}\right)},\left\|D^{2} v_{\epsilon}^{0}\right\|_{L^{\infty}\left(\mathbb{R}^{2}\right)} \leq C$ (see for instance [40]). It can be checked that $\left\|D v_{\epsilon}^{0}\right\|_{L^{\infty}\left(\mathbb{R}^{2}\right)} \leq B_{0}$ and $\left\|D^{2} v_{\epsilon}^{0}\right\|_{L^{\infty}\left(\mathbb{R}^{2}\right)} \leq \frac{B_{0}}{\epsilon} C_{2}$ with $C_{2}$ a positive constant (this calculus is similar to that performed in [40]). Moreover,

$$
\begin{aligned}
\left|v_{0}(x)-v_{\epsilon}^{0}(x)\right| & =\left|v_{0}(x)-\int_{\mathbb{R}^{2}} v_{0}(x-y) \rho_{\epsilon}(y) d y\right| \\
& =\left|\int_{\mathbb{R}^{2}}\left(v_{0}(x)-v_{0}(x-y)\right) \rho_{\epsilon}(y) d y\right|, \\
& \leq \int_{\mathbb{R}^{2}}\left|v_{0}(x)-v_{0}(x-y)\right| \rho_{\epsilon}(y) d y \\
& \leq B_{0} \int_{B(0, \epsilon)}|y| \rho_{\epsilon}(y) d y \\
& \leq \epsilon B_{0} .
\end{aligned}
$$

We denote $v_{\epsilon}$ to be the solution with initial condition $v_{\epsilon}^{0}$. Then, by the comparison principle,

$$
\left\|v_{\epsilon^{\prime}}(\cdot, t)-v_{\epsilon}(\cdot, t)\right\|_{L^{\infty}\left(\mathbb{R}^{2}\right)} \leq\left\|v_{\epsilon^{\prime}}^{0}(\cdot, t)-v_{\epsilon}^{0}(\cdot, t)\right\|_{L^{\infty}\left(\mathbb{R}^{2}\right)} .
$$

Also, $v_{\epsilon}^{0}$ uniformly converges to $v_{0}$ so $\left(v_{\epsilon}^{0}\right)_{\epsilon}$ is a Cauchy sequence. Consequently $\left(v_{\epsilon}(\cdot, t)\right)_{\epsilon}$ is a Cauchy sequence and uniformly converges to $v$ which is, by stability (see [14]), the solution of (2.2) with initial condition $v_{0}$. Still using the comparison principle, we have

Finally,

$$
\left\|v_{\epsilon}(\cdot, t)-v(\cdot, t)\right\|_{L^{\infty}\left(\mathbb{R}^{2}\right)} \leq\left\|v_{\epsilon}^{0}-v_{0}\right\|_{L^{\infty}\left(\mathbb{R}^{2}\right)}
$$

$$
\begin{aligned}
|v(\cdot, t+h)-v(\cdot, t)| & =\left|v(\cdot, t+h)-v_{\epsilon}(\cdot, t+h)+v_{\epsilon}(\cdot, t+h)-v_{\epsilon}(\cdot, t)+v_{\epsilon}(\cdot, t)-v(\cdot, t)\right|, \\
& \leq\left|v(\cdot, t+h)-v_{\epsilon}(\cdot, t+h)\right|+\left|v_{\epsilon}(\cdot, t+h)-v_{\epsilon}(\cdot, t)\right|+\left|v_{\epsilon}(\cdot, t)-v(\cdot, t)\right|, \\
& \leq 2|| v_{\epsilon}^{0}-v_{0} \|_{L^{\infty}\left(\mathbb{R}^{2}\right)}+C_{1}\left(B_{0}, \frac{B_{0}}{\epsilon} C_{2}\right) h . \\
& \leq 2 \epsilon B_{0}+C_{1}\left(B_{0}, \frac{B_{0}}{\epsilon} C_{2}\right) h
\end{aligned}
$$


and

$$
\|v(\cdot, t+h)-v(\cdot, t)\|_{L^{\infty}\left(\mathbb{R}^{2}\right)} \leq 2 \epsilon B_{0}+C_{1}\left(B_{0}, \frac{B_{0}}{\epsilon} C_{2}\right) h .
$$

By taking the minimum on $\epsilon$, we obtain the modulus of continuity of $v$ which depends on $B_{0}$.

CONCLUSION 3.2. We have proved the existence and uniqueness of a viscosity solution of problem (2.2), which is bounded, periodic, continuous on $\mathbb{R}^{2} \times[0, T)$, Lipschitz continuous in space so differentiable almost everywhere, and uniformly continuous in time. We now discretize the evolution equation. In the sequel, we set $\Omega \ni x=\left(x_{1}, x_{2}\right)$.

\section{Experimental results}

Let $\Delta x_{1}$ and $\Delta x_{2}$ be the spatial steps, $\Delta t$ be the time step and $\left(x_{1 i}, x_{2 j}\right)=$ $\left(i \Delta x_{1}, j \Delta x_{2}\right)$ be the grid points, $1 \leq i \leq M$ and $1 \leq j \leq N$. For a function $\Psi: \Omega \rightarrow \mathbb{R}$, let $\Psi_{i j}^{n}=\Psi\left(i \Delta x_{1}, j \Delta x_{2}, n \Delta t\right)$. We define the following finite difference schemes:

$$
\begin{array}{lc}
D^{x_{1}} \Psi_{i, j}^{n}=\frac{\Psi_{i+1, j}^{n}-\Psi_{i-1, j}^{n}}{2 \Delta x_{1}} & D^{x_{2}} \Psi_{i, j}^{n}=\frac{\Psi_{i, j+1}^{n}-\Psi_{i, j-1}^{n}}{2 \Delta x_{2}}, \\
D_{-}^{x_{1}} \Psi_{i, j}^{n}=\frac{\Psi_{i, j}^{n}-\Psi_{i-1, j}^{n}}{\Delta x_{1}} & D_{-}^{x_{2}} \Psi_{i, j}^{n}=\frac{\Psi_{i, j}^{n}-\Psi_{i, j-1}^{n}}{\Delta x_{2}}, \\
D_{+}^{x_{1}} \Psi_{i, j}^{n}=\frac{\Psi_{i+1, j}^{n}-\Psi_{i, j}^{n}}{\Delta x_{1}} & D_{+}^{x_{2}} \Psi_{i, j}^{n}=\frac{\Psi_{i, j+1}^{n}-\Psi_{i, j}^{n}}{\Delta x_{2}}, \\
D^{x_{1} x_{1}} \Psi_{i, j}^{n}=\frac{\Psi_{i+1, j}^{n}-2 \Psi_{i, j}^{n}+\Psi_{i-1, j}^{n}}{\Delta x_{1}^{2}} & D^{x_{2} x_{2}} \Psi_{i, j}^{n}=\frac{\Psi_{i, j+1}^{n}-2 \Psi_{i, j}^{n}+\Psi_{i, j-1}^{n}}{\Delta x_{2}^{2}}, \\
D^{x_{1} x_{2}} \Psi_{i, j}^{n}=\frac{\Psi_{i+1, j+1}^{n}+\Psi_{i-1, j-1}^{n}-\Psi_{i+1, j-1}^{n}-\Psi_{i-1, j+1}^{n}}{4 \Delta x_{1} \Delta x_{2}} .
\end{array}
$$

To discretize (2.2), we use an explicit finite difference scheme as follows. The problem is also complemented by Neumann boundary conditions. For the discretization of the convection component, we refer to [56] or [60] (we have used the notation $\left.d=\left(d_{1}, d_{2}\right)\right)$.

$$
\begin{aligned}
v_{i, j}^{n+1}= & v_{i, j}^{n}+\Delta t b_{i, j}\left(D^{x_{1} x_{1}} v_{i, j}^{n}+D^{x_{2} x_{2}} v_{i, j}^{n}\right) \\
& -\Delta t\left(\max \left(\left(d_{1}\right)_{i, j}, 0\right) D_{-}^{x_{1}} v_{i, j}^{n}+\min \left(\left(d_{1}\right)_{i, j}, 0\right) D_{+}^{x_{1}} v_{i, j}^{n}\right. \\
& \left.+\max \left(\left(d_{2}\right)_{i, j}, 0\right) D_{-}^{x_{2}} v_{i, j}^{n}+\min \left(\left(d_{2}\right)_{i, j}, 0\right) D_{+}^{x_{2}} v_{i, j}^{n}\right) \\
& -\Delta t h_{i, j}+\Delta t \mu \frac{D^{x_{1} x_{1}} v_{i, j}^{n}\left(D^{x_{1}} v_{i, j}^{n}\right)^{2}+2 D^{x_{1}} v_{i, j}^{n} D^{x_{2}} v_{i, j}^{n} D^{x_{1}, x_{2}} v_{i, j}^{n}+D^{x_{2} x_{2}} v_{i, j}^{n}\left(D^{x_{2}} v_{i, j}^{n}\right)^{2}}{\left(D^{x_{1}} v_{i, j}^{n}\right)^{2}+\left(D^{x_{2}} v_{i, j}^{n}\right)^{2}+\epsilon} .
\end{aligned}
$$

4.1. Numerical experimentations of extrapolation. The experiments have been performed on a $2.21 \mathrm{GHz}$ Athlon with $1.00 \mathrm{~GB}$ of RAM. In all our experiments, $\Delta x_{1}=\Delta x_{2}=1$. For each test, we provide a view of the initial gradient vector field $-D g(\|D I\|)$ and a view of the extrapolated vector field.

Our first experimental test figure 4.1 and figure 4.2 is similar to those performed by $\mathrm{Xu}$ and Prince in [64, 65] and by Jifeng et al. in [50]. We computed the GVF 
field, the NGVF field and the gradient vector field obtained with the proposed method for this U-shaped object. The initialization was made either by setting $v_{0} \equiv 0$, or by setting $v_{0} \equiv-g(\|D I\|)$. In all the tests we performed, it does not seem to influence the obtained result. The number of iterations as well as the computational time (order of a second) are similar for the three methods.

Our method qualitatively performs in a way similar to the GVF and the NGVF: we increase the capture range of the gradient vector field and we obtain downward components within the boundary concavity. Nevertheless, contrary to the GVF and NGVF models, the method requires only one unknown.
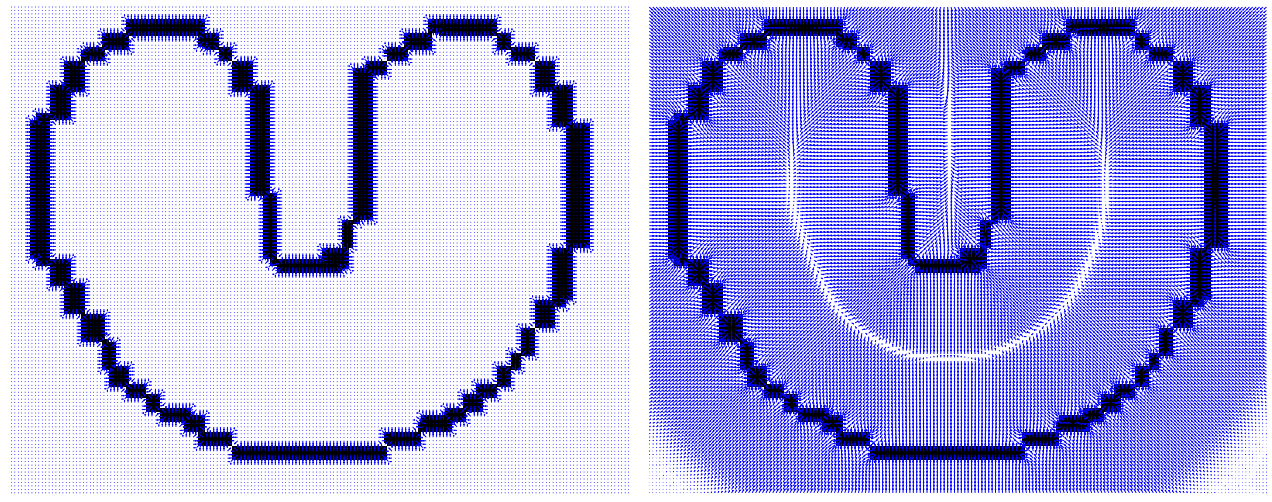

FIG. 4.1. On the left, depiction of the initial gradient vector field $W=-D g(\|D I\|)$. On the right, the obtained vector field with the GVF method ( $\mu=0.1, \Delta t=0.2)$.
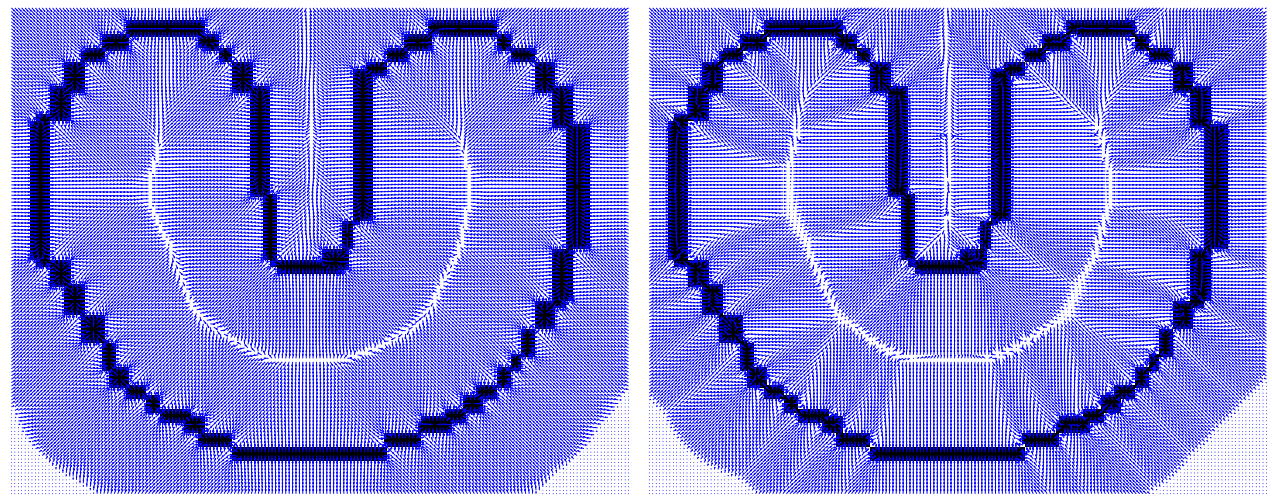

FIG. 4.2. On the left, the obtained vector field with the NGVF method ( $\mu=0.1, \Delta t=0.2)$. On the right, the obtained vector field with our proposed approach $(\mu=0.05, \Delta t=0.1)$.

The second example figure 4.3 and figure 4.4 is also taken from [50] and shows a long and thin concavity. As stressed by Jifeng et al., the NGVF performs more accurately than the GVF in the concavity: the NGVF force field points vertically to the bottom of the concavity, while the GVF fails to enter the concavity. Indeed, the Laplacian operator that naturally appears in the GVF model can be decomposed as the sum of the second derivative in the normal direction, and the second derivative 
in the tangent direction. The former component that is kept in the NGVF model weighs heavily in the extrapolation process and has good properties unlike the later component which proves to be parasitic.

Our proposed approach performs better in the concavity than the GVF model but is not as accurate as the NGVF model. The process seems to be sensitive to the geometry and the thickness of the boundaries.

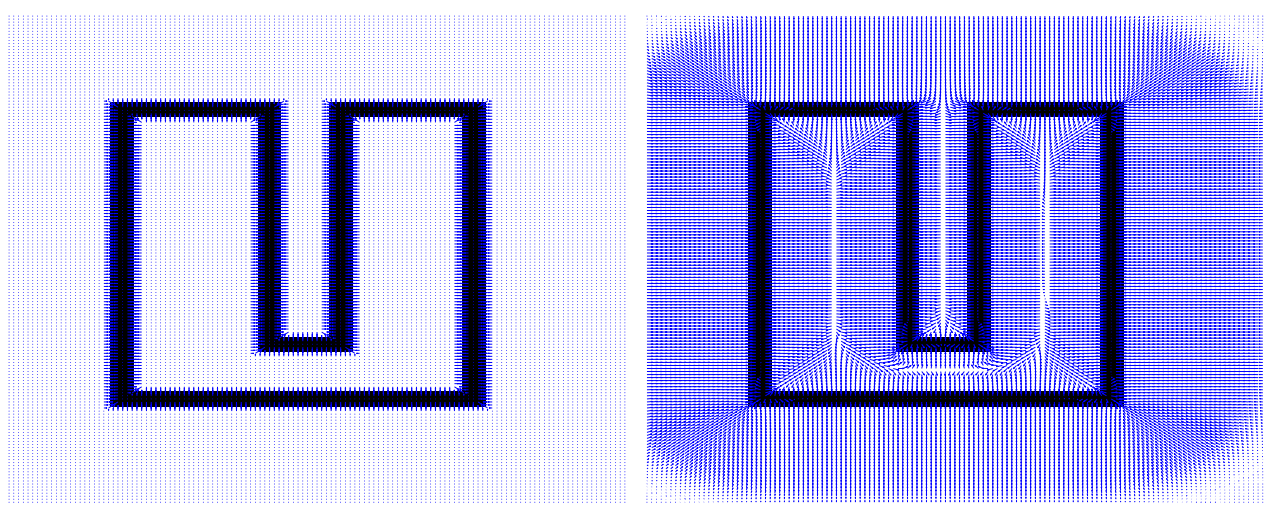

FIG. 4.3. On the left, depiction of the initial gradient vector field $W=-D g(\|D I\|)$. On the right, the obtained vector field with the GVF method $(\mu=0.1, \Delta t=0.2)$.
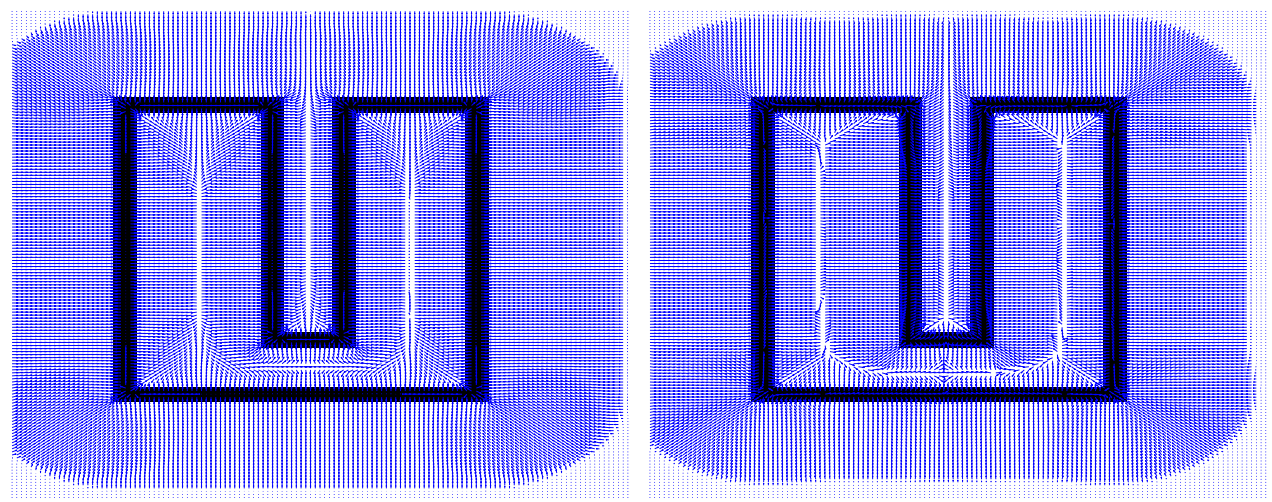

FIG. 4.4. On the left, the obtained vector field with the NGVF method $(\mu=0.1, \Delta t=0.2)$. On the right, the obtained vector field with our proposed approach $(\mu=0.05, \Delta t=0.1)$.

A last example of extrapolation of vector field on a synthetic image is provided in figure 4.5. Finally, we conclude this part with applications to real data. We start with a slice of the brain (figure 4.6, courtesy of the Laboratory of Neuro Imaging, UCLA), with an image taken from the Image Toolbox of Matlab figure 4.7, and with an image showing a slice of Tuffeau (figure 4.8, courtesy of ISTO/ESRF). Our proposed approach performs well but seems to be sensitive to the textures of the objects contained in the image.

4.2. Application to segmentation. This part is dedicated to segmentation and more precisely to the integration of this extrapolated vector field in the geodesic 


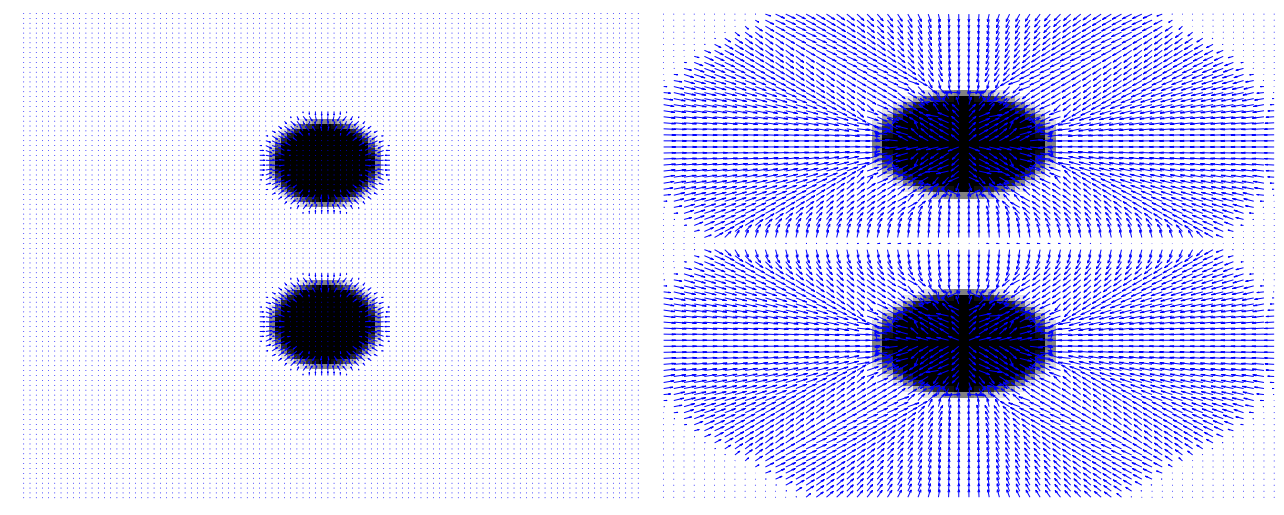

FIG. 4.5. On the left, depiction of the initial gradient vector field $W=-D g(\|D I\|)$. On the right, the obtained vector field with our proposed approach $(\mu=0.05, \Delta t=0.1)$.
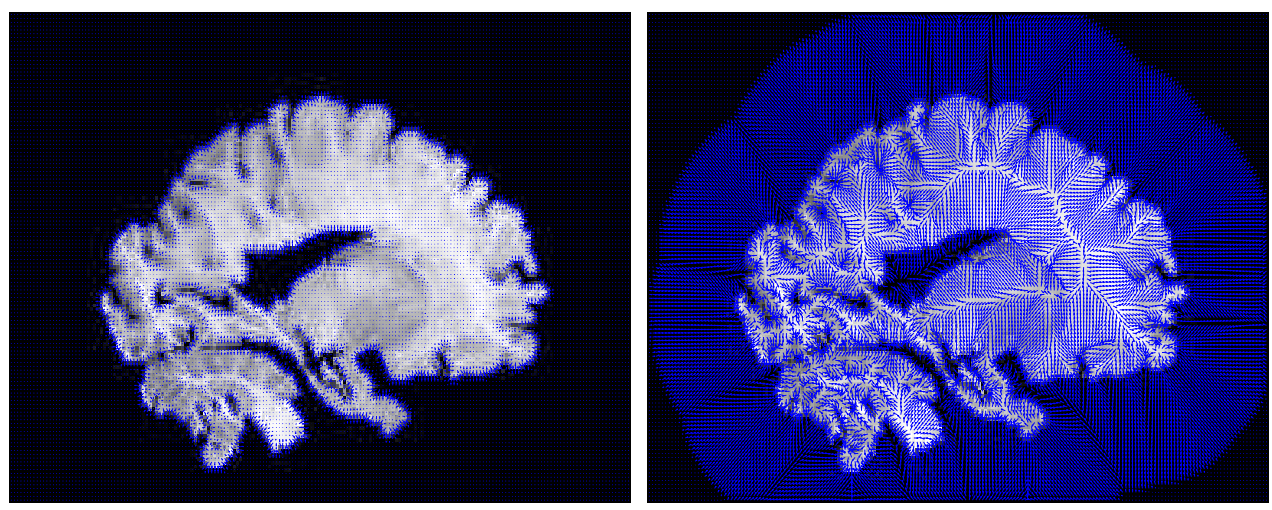

FIG. 4.6. On the left, depiction of the initial gradient vector field $W=-D g(\|D I\|)$. On the right, the obtained vector field with our proposed approach $(\mu=0.05, \Delta t=0.1)$.
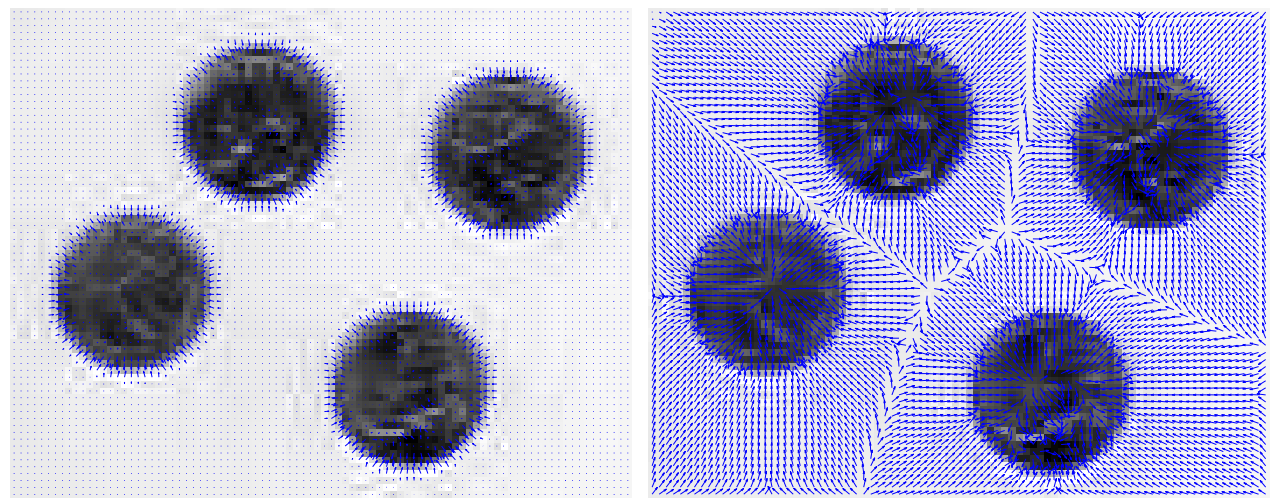

FIG. 4.7. On the left, depiction of the initial gradient vector field $W=-D g(\|D I\|)$. On the right, the obtained vector field with our proposed approach $(\mu=0.05, \Delta t=0.1)$. 

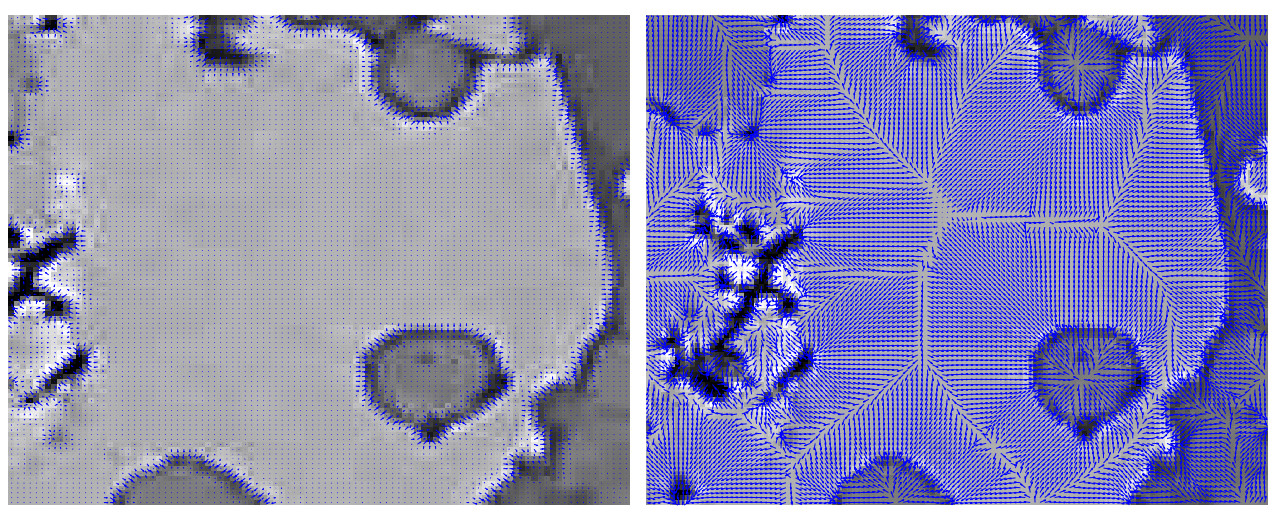

FIG. 4.8. On the left, depiction of the initial gradient vector field $W=-D g(\|D I\|)$. On the right, the obtained vector field with our proposed approach $(\mu=0.1, \Delta t=0.1)$.

active contour model, in order to alleviate the constraint on the choice of the initial condition.

In [28], Caselles et al. introduce the geodesic active contour model. Denoting $\mathcal{C}$ to be a parameterized curve defined by

$$
\mathcal{C}:\left\{\begin{array}{l}
{[0,1] \rightarrow \mathbb{R}^{2}} \\
q \mapsto \mathcal{C}(q),
\end{array}\right.
$$

they propose to minimize the following functional:

$$
\int_{0}^{1} g(\|D I(\mathcal{C}(q))\|)\left\|\mathcal{C}^{\prime}(q)\right\| d q
$$

where $I$ is the image and $g$ is an edge-detector function. The evolution equation of the curve $\mathcal{C}$ to deform the initial contour $\mathcal{C}(., 0)$ towards a local minimizer of (4.2) is established thanks to the Euler-Lagrange theorem and the gradient descent method. One obtains:

$$
\frac{\partial \mathcal{C}}{\partial t}=g(\|D I(\mathcal{C}(q, t))\|) \kappa(q, t) \vec{n}(q, t)-\langle D g(\|D I\|)(\mathcal{C}(q, t)), \vec{n}(q, t)\rangle \vec{n}(q, t),
$$

with $\kappa(q, t)$ the curvature at point $\mathcal{C}(q, t)$ and $\vec{n}(q, t)$ the unit inward normal vector to the curve at point $\mathcal{C}(q, t)$.

In order to avoid certain local minima or to increase the speed of convergence, a constant motion term of the form $c g(\|D I(\mathcal{C}(q, t))\|) \vec{n}(q, t)$ (with $c$ a parameter) can be incorporated into the partial differential equation.

The model is then cast in the level set setting developed by Osher and Sethian in [57]. That is, the evolving curve $\mathcal{C}$ is represented implicitly via an explicit $3 \mathrm{D}$ function denoted by $\Phi$ and at each time $t$, the curve $\mathcal{C}$ is considered as the zero level set of the function $\Phi$ which is a Lipschitz continuous function. This representation is intrinsic and allows splits and merges. We obtain the following partial differential equation which constitutes the general geodesic active contour model:

$$
\frac{\partial \Phi}{\partial t}=\|D \Phi\| \operatorname{div}\left(g(\|D I\|) \frac{D \Phi}{\|D \Phi\|}\right)+c g(\|D I\|)\|D \Phi\|,
$$


that is,

$$
\frac{\partial \Phi}{\partial t}=g(\|D I\|)\|D \Phi\| \operatorname{div}\left(\frac{D \Phi}{\|D \Phi\|}\right)+\langle D g(\|D I\|), D \Phi\rangle+c g(\|D I\|)\|D \Phi\| .
$$

The component $\langle D g(\|D I\|), D \Phi\rangle$ guarantees a good attraction towards the boundaries one aims at detecting but acts locally in a narrow band around the edges. We propose, as done in [58], to replace $W=-D g(\|D I\|)$ by the extrapolated vector field obtained with our proposed approach.

To illustrate this, we propose an example which still uses the two disks in figure 4.9. In this example, we aim at demonstrating that the initialization stage is now more flexible than in the case of the classical geodesic active contour model. The initial condition is made of two contours: one inside the upper disk, the other enclosing the lower disk. Of course, with an initial contour enclosing both disks, or composed of two contours enclosing each disk or initialized inside each disk, the extrapolation stage would not be necessary and the classical geodesic active contour model would perform adequately. But with this initialization, even with the inflation/deflation force, we are not guaranteed that the classical geodesic active contour model will provide the expected segmentation: it will more probably fail to detect both shapes.

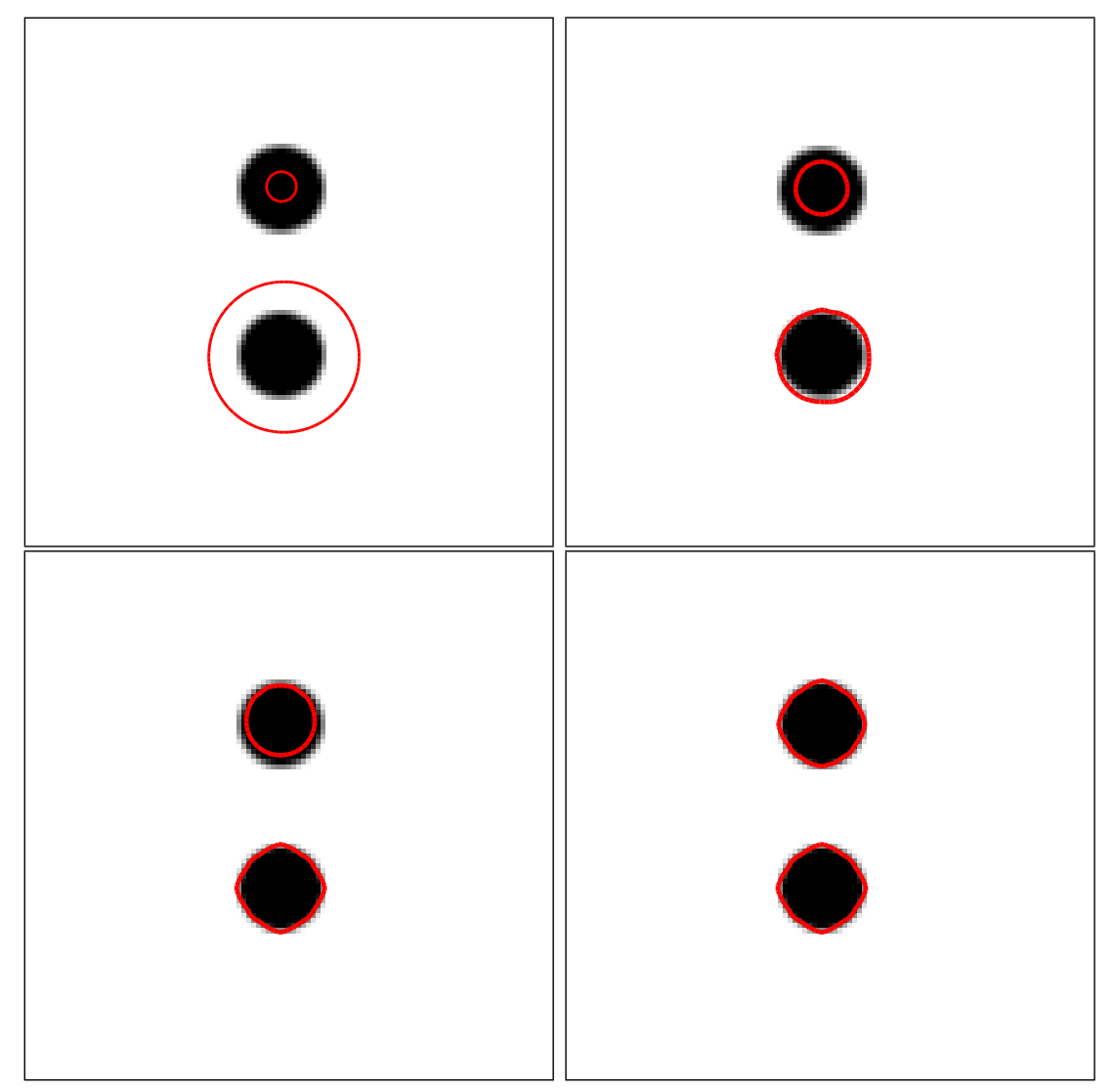

FIG. 4.9. Steps of the segmentation of the synthetic image with two disks (200 iterations).

We propose, for this example, a comparison figure 4.10 with another method [41] 
based on the Generalized Fast Marching Method (GFMM) (see [23]), and that allows weakening of the constraint in the initialization stage. The GFMM developed by Carlini et al. is an extension of the Fast Marching Method (FMM) that removes the sign constraint (the normal velocity is of constant sign in the case of the FMM) by authorizing time-dependent velocity with no restriction on the sign. The obtained results are similar (computation time of the same order) but the obtained contour is more regular in the case of the proposed method. Nevertheless, in more general cases the GFMM-based method offers more flexibility in the initialization stage (see [41] for more details). A second example taken from [58] is given in figure 4.11. This synthetic

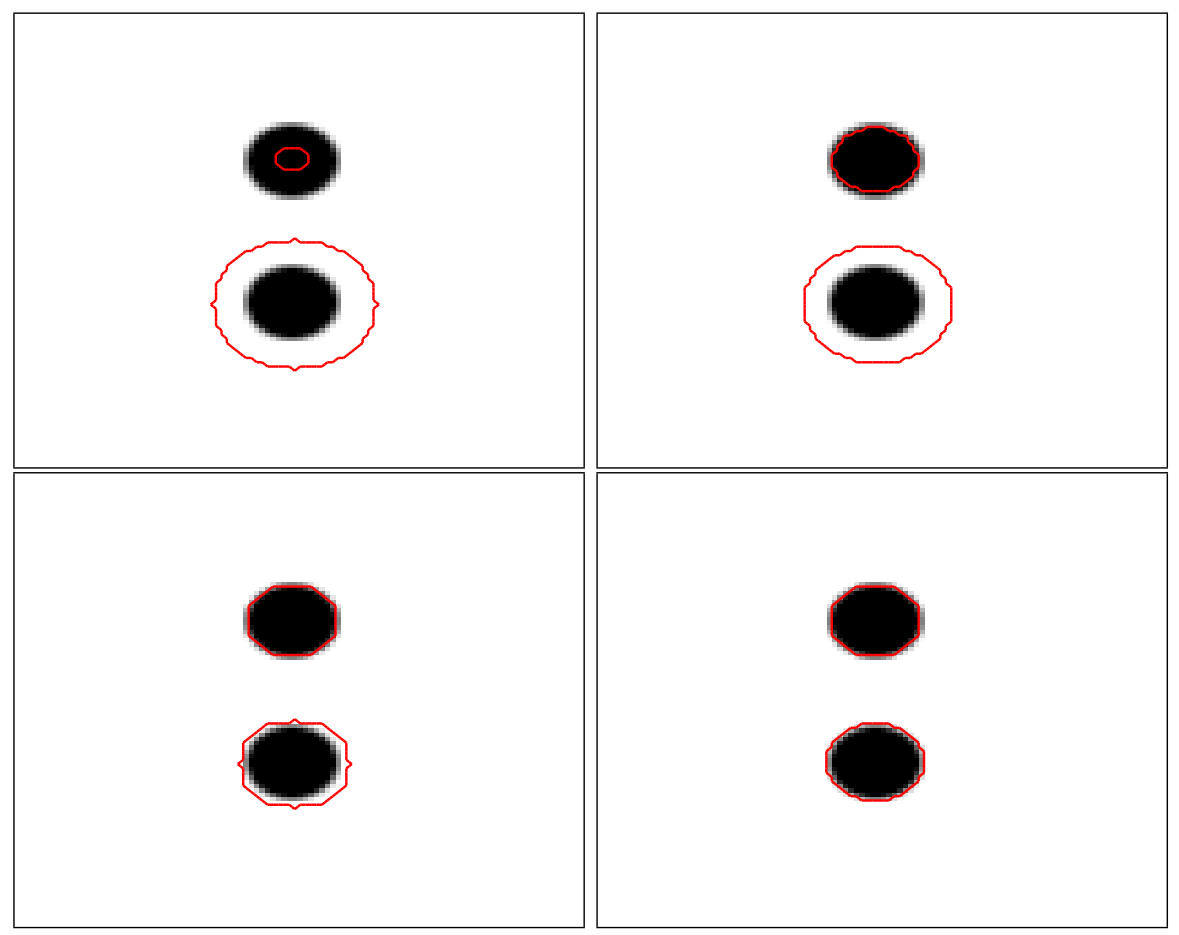

FIG. 4.10. Steps of the segmentation of the synthetic image with two disks by the method developed in [41].

image contains convex geometrical shapes. The representation of the extrapolated vector field (not given here) shows that the vector flow points towards the boundaries of the closest object. This example demonstrates that the initial condition (composed of several shapes here) can be made inside, outside or across the boundaries, provided the initial curves contain part of the extrapolated vector flow skeleton. Of course, the proposed method cannot detect automatically interior contours but this drawback is overcome by the flexibility in the initialization step. We illustrate this remark with the example proposed in figure 4.12 (similar to the one in figure 4.6) by taking as initial shape a deformed torus. This would not be possible with the application of the classical geodesic active contour method. To finish, we propose an illustration on a sequence of images representing cross-sections of the main pulmonary artery (courtesy of CHU du Haut-Lévêque, Pessac) (see figure 4.13). The quality of the first image of the sequence is moderate. The contrast between the vessel wall and the surrounding 

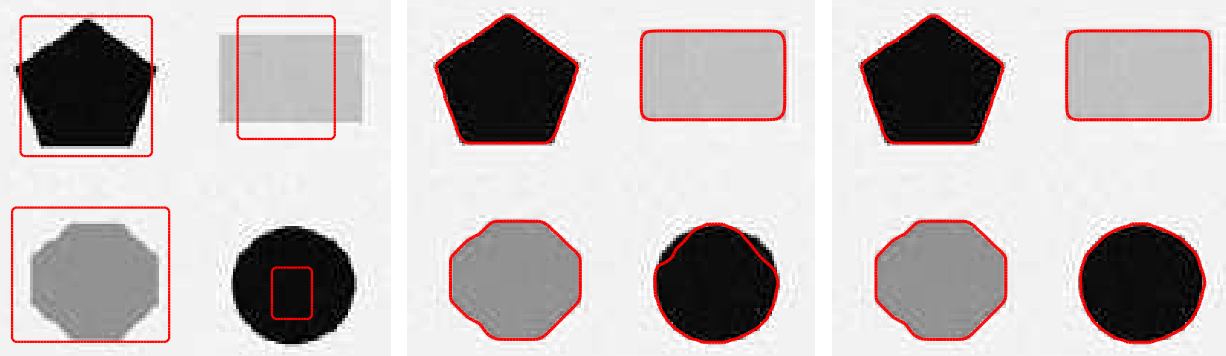

FIG. 4.11. Steps of the segmentation of the synthetic image taken from [58].
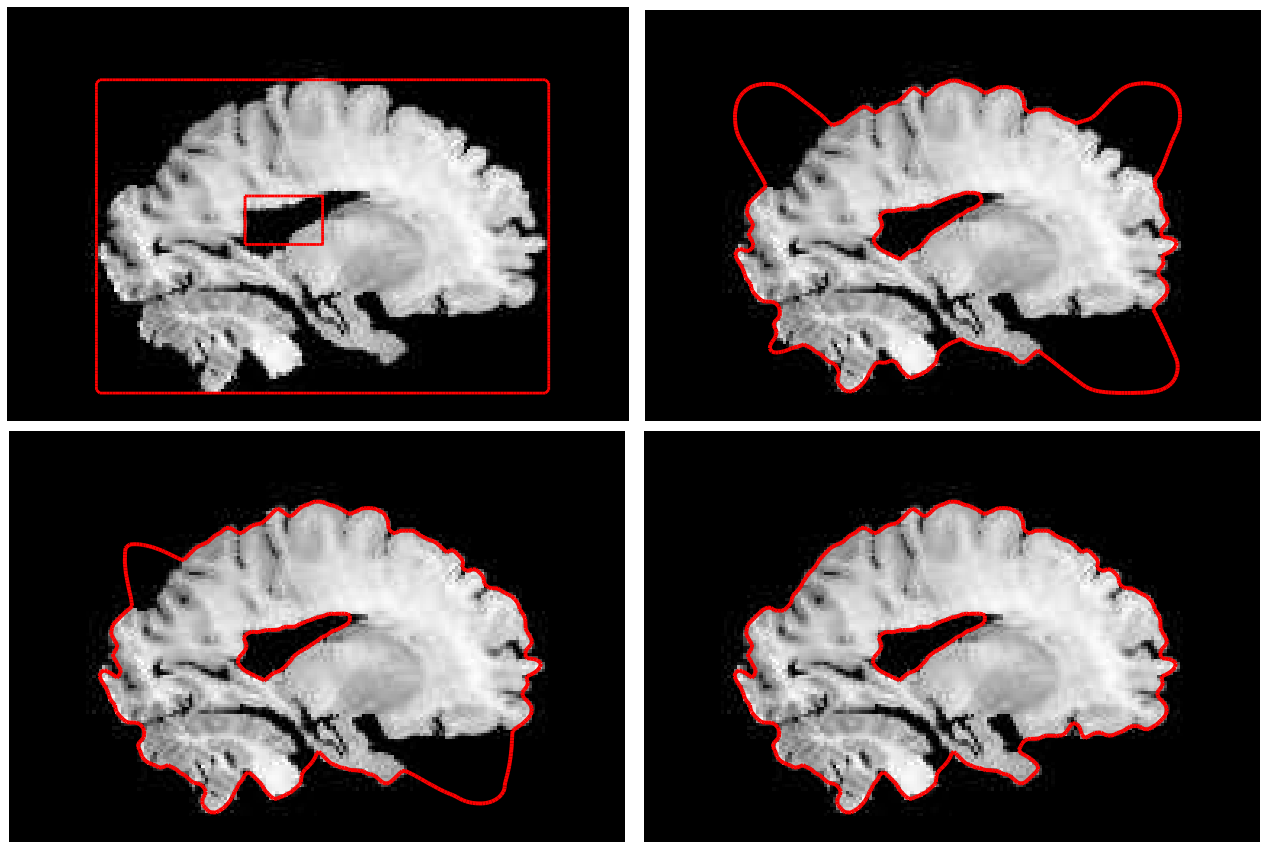

FIG. 4.12. Steps of the segmentation process of a slice of the brain.

medium (see figure 4.14) is impaired at the bottom left-hand side of the section. Impairment in the contrast can be related to partial volume effect, flow turbulences, that are correlated with the severity of the pulmonary arterial hypertension, and the absence of the so-called 'proton inflow phenomenon', which leads to poor blood image contrast at the end-diastolic cardiac phase in the image frame.

To cope with this difficulty, we propose to apply our algorithm to increase the capture range of the external force field. The initial condition (see figure 4.15) crosses the object boundary. The obtained final contour for the first image is then used as an initial condition for the second image figure 4.16, and so on (see figure 4.17, figure 4.18 and figure 4.19). All the images are segmented according to the same process. The time step is fixed at $\Delta t=0.2$, and the coefficient balancing the component related to the extrapolated vector field is chosen equal to 2.0 . 

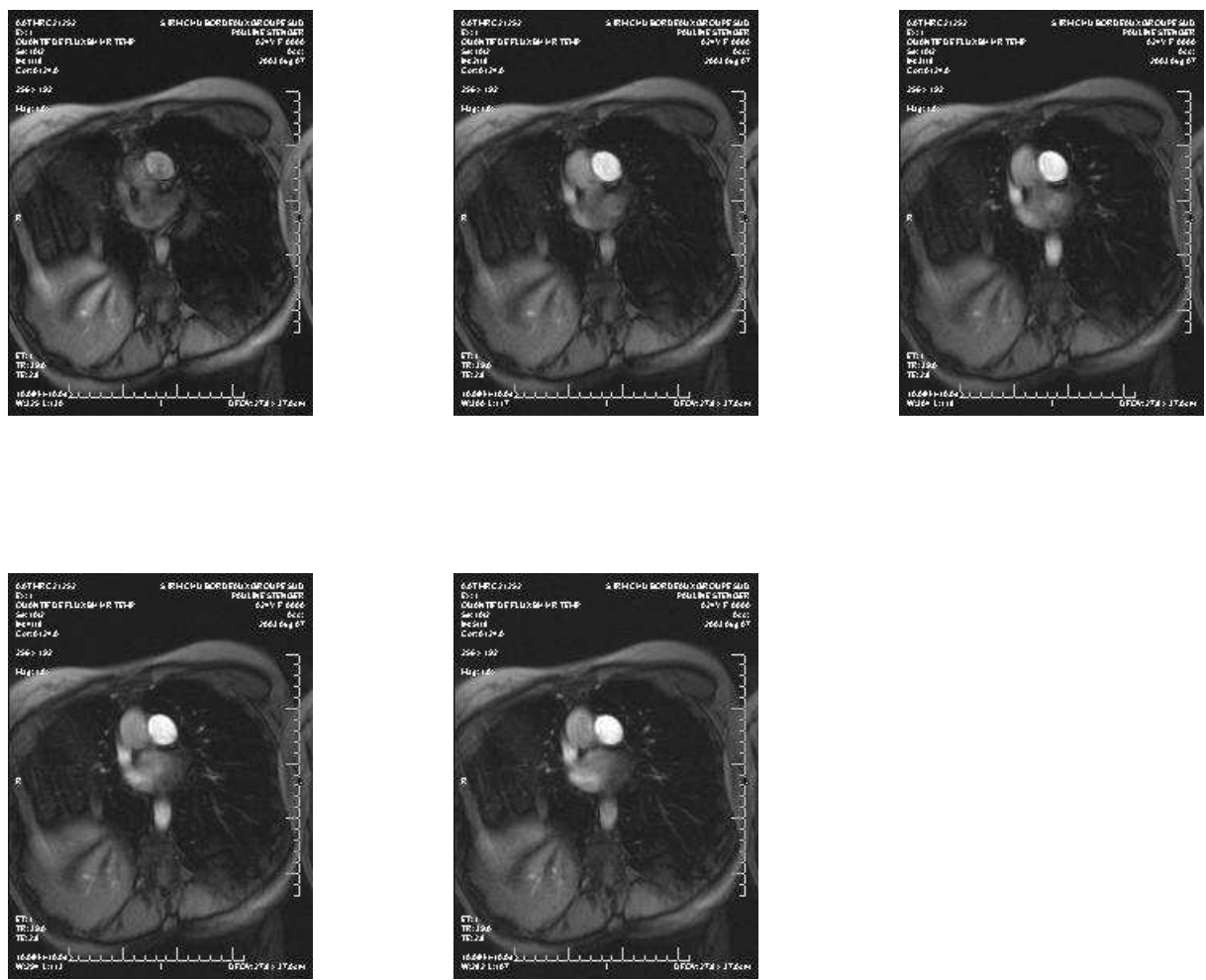

FIG. 4.13. Cross-sections of the main pulmonary artery.

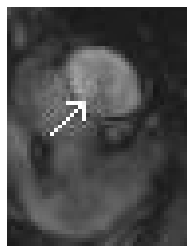

FIG. 4.14. Zoom of the portion of the pulmonary artery section that exhibits weak edge.

\section{Conclusion}

This paper was devoted to the theoretical study of a new method to extrapolate vector fields using the infinity Laplacian and with applications to image processing. Contrary to prior related works, the number of unknowns is reduced to a single one. The problem is phrased in a variational framework and the Euler-Lagrange equation is then derived. It is solved using a gradient descent method, which leads to a parabolic problem that falls within the viscosity solution theory framework.

The existence and uniqueness of a viscosity solution continuous in space and time, Lipschitz continuous in space and uniformly continuous in time is established.

The theoretical study is complemented by several numerical experiments, first dedicated to the extrapolation problem, and then extended to the segmentation problem. 

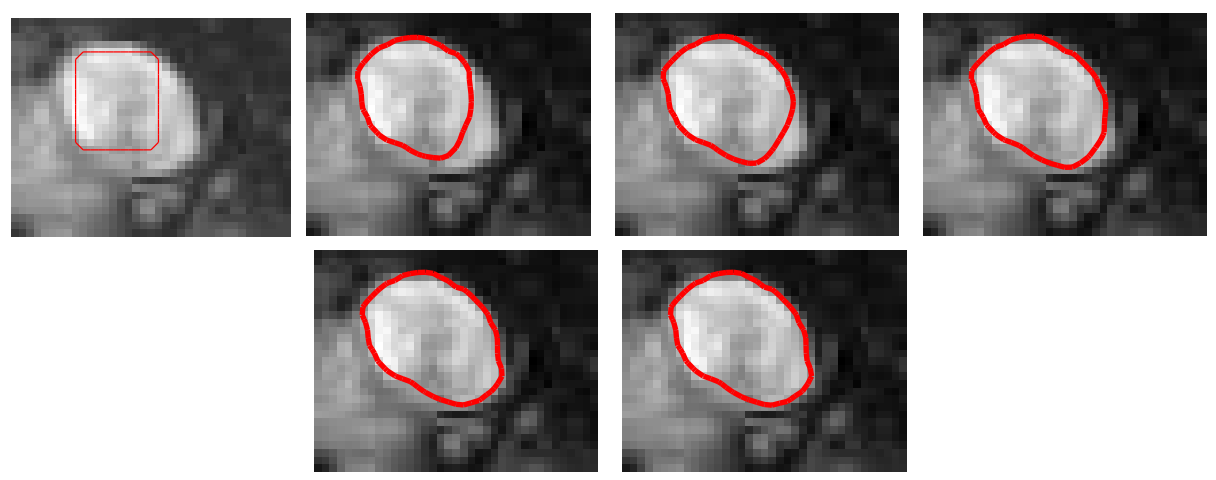

FIG. 4.15. Steps of the segmentation process for the first image of the sequence (iterations 10, 20, 30, 40, 50).
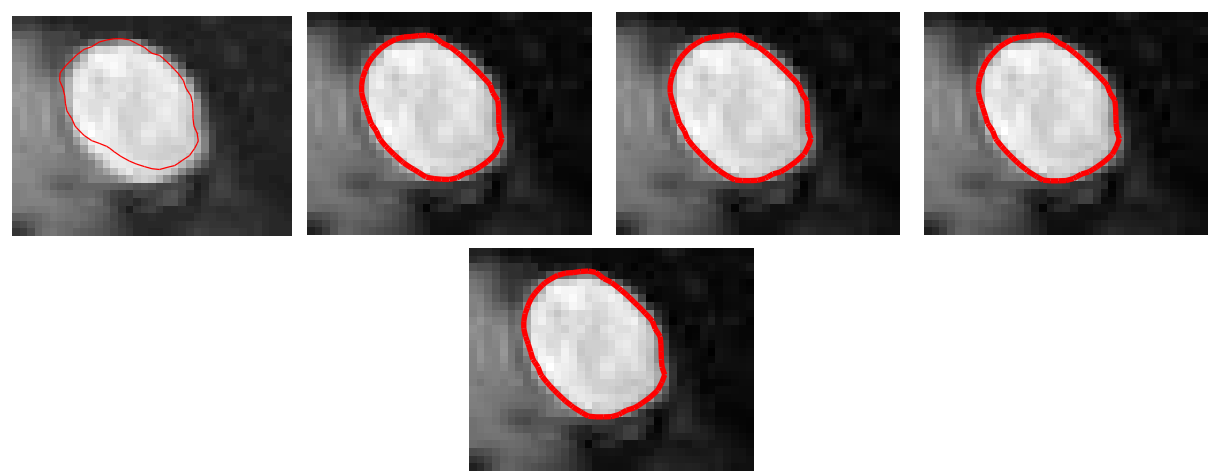

FIG. 4.16. Steps of the segmentation process for the second image of the sequence (iterations $10,20,30,40$ ).
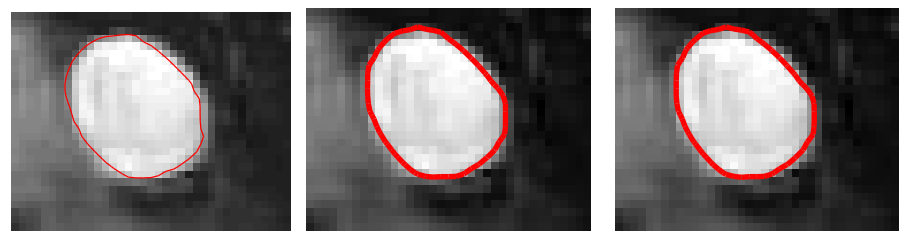
20).

FIG. 4.17. Steps of the segmentation process for the third image of the sequence (iterations 10,
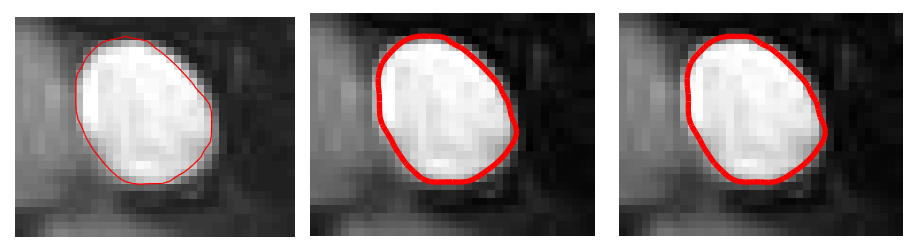

FIG. 4.18. Steps of the segmentation process for the fourth image of the sequence (iterations 10, 20). 

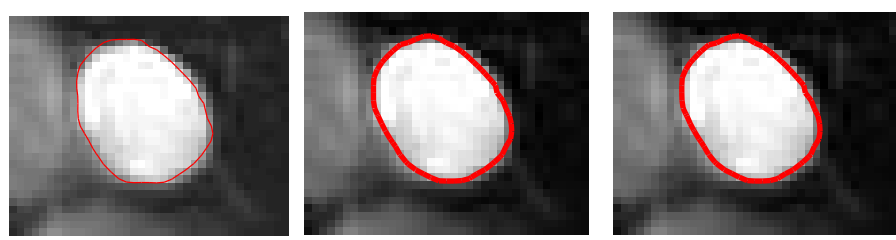

FIG. 4.19. Steps of the segmentation process for the last image of the sequence (iterations 10, 20).

The experiments show that the proposed approach performs well, even if in strong concavities the results are slightly less accurate than with the NGVF. The model is sensitive to the geometry of the boundaries and to the textures present in the images.

In the segmentation framework, the introduction of this new force field allows to widen the choice of the initial condition.

Acknowledgement. The authors would like to thank Nicolas Forcadel for fruitful, enriching and stimulating discussions on this project. Also, the authors would like to thank Luminita Vese for suggesting some references on AMLE and on applications to image processing, and for her very helpful comments. The authors are very grateful to the unknown referees for their careful reading of the manuscript and for their comments which were very helpful in preparing the final improved version of the paper. To finish, the first author would like to thank Dominique Apprato for arousing her interest in the theory of approximation and for the enlighting discussions they have had on this subject.

Appendix A. Main steps of Proof 3. For the sake of clarity, in this proof we denote $\Psi$ to be the initial condition of the evolution problem $(2.2)$, that is, $v(x, 0)=$ $\Psi(x)$ in $(2.2)$.

We follow the arguments of [33]. We first observe that for $\lambda>0, \tilde{u}=u-\frac{\lambda}{T-t}$ is also a subsolution of $(2.2)$ and

$$
\tilde{u}_{t}+G_{*}\left(x, D \tilde{u}, D^{2} \tilde{u}\right) \leq-\frac{\lambda}{(T-t)^{2}} \leq-\frac{\lambda}{T^{2}} .
$$

Since $u \leq v$ follows from $\tilde{u} \leq v$ in the limit $\lambda \rightarrow 0$, it will simply suffice to prove the comparison under the additional assumptions:

$$
\left\{\begin{array}{l}
\text { (i) } u_{t}+G_{*}\left(x, D u, D^{2} u\right) \leq-\frac{\lambda}{T^{2}}, \\
\text { (ii) } \lim _{t \rightarrow T} u(x, t)=-\infty
\end{array}\right.
$$

Let us set

$$
M=\sup _{\mathbb{R}^{2} \times[0, T)} u(x, t)-v(x, t) .
$$

We aim to show that $M \leq 0$. We argue by contradiction and assume that $M>0$.

Let us also introduce $M_{0}$ defined by:

$$
M_{0}=\sup _{\mathbb{R}^{2} \times \mathbb{R}^{2} \times[0, T)}\left\{u(x, t)-v(y, t)-(4 \epsilon)^{-1}|x-y|^{4}\right\}, \epsilon>0 .
$$


This supremum is reached, owing to the bound above of $u$ and $-v$, the fact that $f(x, y, t)=u(x, t)-v(y, t)-(4 \epsilon)^{-1}|x-y|^{4}$ is such that $\forall h \in \mathbb{Z}^{2}, f(x+2 h, y+2 h, t)=$ $f(x, y, t)$, and (A.1)(ii). We denote $\left(x_{0}, y_{0}, t_{0}\right) \in \mathbb{R}^{2} \times \mathbb{R}^{2} \times[0, T)$ to be a maximum point. Consequently, $x_{0}, y_{0}$ and $t_{0}$ depend on $\epsilon$ and $M_{0}=u\left(x_{0}, t_{0}\right)-v\left(y_{0}, t_{0}\right)-$ $(4 \epsilon)^{-1}\left|x_{0}-y_{0}\right|^{4}$. Obviously, $M_{0} \geq M>0$.

Let us assume that $t_{0}=0$. Because the function $u$ is a subsolution, we have $u(x, 0) \leq \Psi(x)$ on $\mathbb{R}^{2}$. Similarly, because the function $v$ is a supersolution, we have $v(x, 0) \geq \Psi(x)$ on $\mathbb{R}^{2}$.

Consequently,

$$
\begin{aligned}
M & \leq M_{0}=u\left(x_{0}, 0\right)-v\left(y_{0}, 0\right)-(4 \epsilon)^{-1}\left|x_{0}-y_{0}\right|^{4} \leq \Psi\left(x_{0}\right)-\Psi\left(y_{0}\right)-(4 \epsilon)^{-1}\left|x_{0}-y_{0}\right|^{4}, \\
& \leq \sup _{\mathbb{R}^{2} \times \mathbb{R}^{2}}\left(\Psi(x)-\Psi(y)-(4 \epsilon)^{-1}|x-y|^{4}\right) .
\end{aligned}
$$

We first observe that $0 \leq \sup _{\mathbb{R}^{2} \times \mathbb{R}^{2}}\left(\Psi(x)-\Psi(y)-(4 \epsilon)^{-1}|x-y|^{4}\right)$. Using the same arguments as those previously mentioned, this supremum is reached. We denote $\left(x_{\epsilon}, y_{\epsilon}\right)$ to be a maximum point of $\Psi(x)-\Psi(y)-(4 \epsilon)^{-1}|x-y|^{4}$. Denoting by $R=\|\Psi\|_{L^{\infty}\left(\mathbb{R}^{2}\right)}$, we then obtain

$$
0 \leq \Psi\left(x_{\epsilon}\right)-\Psi\left(y_{\epsilon}\right)-(4 \epsilon)^{-1}\left|x_{\epsilon}-y_{\epsilon}\right|^{4} \leq 2 R-(4 \epsilon)^{-1}\left|x_{\epsilon}-y_{\epsilon}\right|^{4},
$$

so $\left|x_{\epsilon}-y_{\epsilon}\right|^{4} \leq 8 \epsilon R$ and $\left|x_{\epsilon}-y_{\epsilon}\right| \leq(8 \epsilon R)^{\frac{1}{4}}$.

But, $0 \leq \Psi\left(x_{\epsilon}\right)-\Psi\left(y_{\epsilon}\right)-(4 \epsilon)^{-1}\left|x_{\epsilon}-y_{\epsilon}\right|^{4} \leq B_{0}\left|x_{\epsilon}-y_{\epsilon}\right|\left(B_{0}\right.$ Lipschitz constant of $\left.\Psi\right)$.

Thus passing to the limit in (A.2) when $\epsilon$ tends to 0 , it yields that $\sup _{\mathbb{R}^{2} \times \mathbb{R}^{2}}(\Psi(x)-$ $\left.\Psi(y)-(4 \epsilon)^{-1}|x-y|^{4}\right) \underset{\epsilon \rightarrow 0}{\longrightarrow} 0$, which contradicts $M>0$. So $t_{0}>0$ if $\epsilon$ is small enough.

In the following, we denote by $\varphi$ the function defined by $\varphi(x, y, t)=(4 \epsilon)^{-1}|x-y|^{4}$. We now distinguish two cases:

$\underline{\text { Case } x_{0}=y_{0}:}$

By virtue of Theorem 8.3 from [33], setting $\tau=\partial_{t} \varphi\left(x_{0}, y_{0}, t_{0}\right)=0$ and $A=$ $D^{2} \varphi\left(x_{0}, y_{0}, t_{0}\right)$, for all $\gamma>0$ there exist $\tau_{1}, \tau_{2} \in \mathbb{R}$ and $X, Y \in \mathcal{S}^{2}$ such that

$$
\begin{array}{r}
\tau=0=\tau_{1}-\tau_{2}, \\
\left(\tau_{1}, p_{1}, X\right) \in \overline{\mathcal{P}}^{+} u\left(x_{0}, t_{0}\right),\left(\tau_{2}, p_{2}, Y\right) \in \overline{\mathcal{P}}^{-} v\left(y_{0}, t_{0}\right), \\
-\left(\frac{1}{\gamma}+\|A\|\right) I \leq\left(\begin{array}{cc}
X & 0 \\
0 & -Y
\end{array}\right) \leq A+\gamma A^{2},
\end{array}
$$

with $p_{1}=D_{x} \varphi\left(x_{0}, y_{0}, t_{0}\right)$ and $p_{2}=-D_{y} \varphi\left(x_{0}, y_{0}, t_{0}\right)$. In the following, we set $p_{0}=x_{0}-$ $y_{0}$.

A mere calculus gives $p_{1}=(\epsilon)^{-1}\left|x_{0}-y_{0}\right|^{2}\left(x_{0}-y_{0}\right)=(\epsilon)^{-1}\left|p_{0}\right|^{2} p_{0}, p_{2}=p_{1}$, and

$$
A=\frac{2}{\epsilon}\left|p_{0}\right|^{2}\left(\begin{array}{cc}
Z & -Z \\
-Z & Z
\end{array}\right), \text { with } Z=\frac{I}{2}+\frac{p_{0} \otimes p_{0}}{\left|p_{0}\right|^{2}}
$$


This last inequality gives that $X \leq Y$, owing to the particular form of matrix $A$. A:

Lemma A.1 (Matrix estimates.). We have the following estimates on the matrix

$$
\left\{\begin{array}{l}
\|A\| \leq \frac{6\left|p_{0}\right|^{2}}{\epsilon}, \\
A \leq\|A\| I \text { and } \frac{1}{\|A\|} A^{2} \leq\|A\| I,
\end{array}\right.
$$

where $\|A\|=\sup _{\xi \in \mathbb{R}^{4} \backslash\left\{0_{\mathbb{R}^{4}}\right\}} \frac{\langle A \xi, \xi\rangle}{\langle\xi, \xi\rangle}$.

Proof. Let us set $\xi=\left(\begin{array}{l}\xi_{1} \\ \xi_{2}\end{array}\right) \in \mathbb{R}^{4}$.

$$
\begin{aligned}
\langle A \xi, \xi\rangle & =\frac{2\left|p_{0}\right|^{2}}{\epsilon}\left\langle Z\left(\xi_{1}-\xi_{2}\right), \xi_{1}-\xi_{2}\right\rangle, \\
& \leq \frac{2\left|p_{0}\right|^{2}}{\epsilon}\|Z\|\left|\xi_{1}-\xi_{2}\right|^{2} \\
& \leq \frac{4\left|p_{0}\right|^{2}}{\epsilon}\|Z\||\xi|^{2} .
\end{aligned}
$$

So, $\|A\| \leq \frac{4\left|p_{0}\right|^{2}}{\epsilon}\|Z\|$. Moreover, now with $\xi=\left(\begin{array}{l}\xi_{1} \\ \xi_{2}\end{array}\right) \in \mathbb{R}^{2}$,

$$
\begin{aligned}
\langle Z \xi, \xi\rangle & =\frac{1}{2}|\xi|^{2}+\left\langle\frac{p_{0} \otimes p_{0}}{\left|p_{0}\right|^{2}} \xi, \xi\right\rangle, \\
& =\frac{1}{2}|\xi|^{2}+\frac{1}{\left|p_{0}\right|^{2}}\left\langle p_{0}, \xi\right\rangle^{2}, \\
& \leq \frac{1}{2}|\xi|^{2}+|\xi|^{2}=\frac{3}{2}|\xi|^{2} .
\end{aligned}
$$

We finally deduce that $\|Z\| \leq \frac{3}{2}$ and $\|A\| \leq \frac{6}{\epsilon}\left|p_{0}\right|^{2}$.

Let us take $\xi=\left(\begin{array}{l}\xi_{1} \\ \xi_{2}\end{array}\right) \in \mathbb{R}^{4}$.

$$
\langle A \xi, \xi\rangle \leq\|A\||\xi|^{2}=\|A\|\langle I \xi, \xi\rangle \quad \text { and } \quad \frac{1}{\|A\|}\left\langle A^{2} \xi, \xi\right\rangle \leq\|A\||\xi|^{2}=\|A\|\langle I \xi, \xi\rangle \text {. }
$$

Using the previous lemma and taking $\gamma=\frac{1}{\|A\|}$, we can rewrite the matrix inequality (A.3) in the following form:

$$
-\frac{12}{\epsilon}\left|p_{0}\right|^{2} I \leq\left(\begin{array}{cc}
X & 0 \\
0 & -Y
\end{array}\right) \leq \frac{12}{\epsilon}\left|p_{0}\right|^{2} I .
$$

Because the function $u$ is a subsolution and the function $v$ is a supersolution, we have, using (A.1)(i),

$$
\begin{aligned}
& \tau_{1}+c\left(x_{0},(\epsilon)^{-1}\left|p_{0}\right|^{2} p_{0}\right)+E\left(x_{0}, X\right)+F_{*}\left((\epsilon)^{-1}\left|p_{0}\right|^{2} p_{0}, X\right) \leq-\frac{\lambda}{T^{2}} \\
& \tau_{2}+c\left(y_{0},(\epsilon)^{-1}\left|p_{0}\right|^{2} p_{0}\right)+E\left(y_{0}, Y\right)+F^{*}\left((\epsilon)^{-1}\left|p_{0}\right|^{2} p_{0}, Y\right) \geq 0
\end{aligned}
$$


If $x_{0}=y_{0}$, from (A.4), it yields $X=0$ and $Y=0$. Having $F^{*}(0,0)=F_{*}(0,0)=0$ and $E(x, 0)=0$, we thus obtain from (A.5) that $\frac{\lambda}{T^{2}} \leq 0$, which is absurd.

Case $x_{0} \neq y_{0}$ :

$\forall \gamma>0$, we have $\left(\begin{array}{cc}X & 0 \\ 0 & -Y\end{array}\right) \leq A+\gamma A^{2}$. As $x_{0} \neq y_{0}$, we take $\gamma=\frac{1}{2} \epsilon\left|p_{0}\right|^{-2}$ and obtain

$$
\left(\begin{array}{cc}
X & 0 \\
0 & -Y
\end{array}\right) \leq 2 \epsilon^{-1}\left(\begin{array}{cc}
C & -C \\
-C & C
\end{array}\right)
$$

with $C=\left|p_{0}\right|^{2} I+5 p_{0} \otimes p_{0}$. From this last inequality, it yields $X \leq Y$ and using the ellipticity of $F$, the following holds:

$$
F\left((\epsilon)^{-1}\left|p_{0}\right|^{2} p_{0}, Y\right)-F\left((\epsilon)^{-1}\left|p_{0}\right|^{2} p_{0}, X\right) \leq 0 .
$$

$E\left(y_{0}, Y\right)-E\left(x_{0}, X\right)=b\left(x_{0}\right) \operatorname{trace}(X)-b\left(y_{0}\right) \operatorname{trace}(Y)$,

$$
\begin{aligned}
& =\operatorname{trace}\left(\begin{array}{cc}
b\left(x_{0}\right) X & 0 \\
0 & -b\left(y_{0}\right) Y
\end{array}\right), \\
& =\operatorname{trace}\left(\left(\begin{array}{cc}
b\left(x_{0}\right) I & \sqrt{b\left(x_{0}\right) b\left(y_{0}\right)} I \\
\sqrt{b\left(x_{0}\right) b\left(y_{0}\right)} & b\left(y_{0}\right) I
\end{array}\right)\left(\begin{array}{cc}
X & 0 \\
0 & -Y
\end{array}\right)\right)=\operatorname{trace}\left(G\left(\begin{array}{cc}
X & 0 \\
0 & -Y
\end{array}\right)\right),
\end{aligned}
$$

where $G$ is the matrix defined by $G=\left(\begin{array}{cc}b\left(x_{0}\right) I & \sqrt{b\left(x_{0}\right) b\left(y_{0}\right)} I \\ \sqrt{b\left(x_{0}\right) b\left(y_{0}\right)} I & b\left(y_{0}\right) I\end{array}\right)$.

Because the matrix $G$ is symmetric semipositive definite, it can be written in the form of $G=\chi \chi^{T}$ and

$$
\begin{aligned}
\operatorname{trace}\left(G\left(\begin{array}{cc}
X & 0 \\
0 & -Y
\end{array}\right)\right) & =\operatorname{trace}\left(\chi \chi^{T}\left(\begin{array}{cc}
X & 0 \\
0 & -Y
\end{array}\right)\right)=\operatorname{trace}\left(\chi^{T}\left(\begin{array}{cc}
X & 0 \\
0 & -Y
\end{array}\right) \chi\right), \\
& =\sum_{i=1}^{4} \chi_{i}^{T}\left(\begin{array}{cc}
X & 0 \\
0 & -Y
\end{array}\right) \chi_{i},
\end{aligned}
$$

where $\chi_{i}$ is the $\mathrm{i}^{\text {th }}$ column of $\chi$. Using inequality (A.6), we have

$$
\sum_{i=1}^{4} \chi_{i}^{T}\left(\begin{array}{cc}
X & 0 \\
0 & -Y
\end{array}\right) \chi_{i} \leq 2 \epsilon^{-1} \sum_{i=1}^{4} \chi_{i}^{T}\left(\begin{array}{cc}
C & -C \\
-C & C
\end{array}\right) \chi_{i}
$$

so,

$$
\begin{aligned}
\operatorname{trace}\left(G\left(\begin{array}{cc}
X & 0 \\
0 & -Y
\end{array}\right)\right) & \leq 2 \epsilon^{-1} \operatorname{trace}\left(G\left(\begin{array}{cc}
C & -C \\
-C & C
\end{array}\right)\right) \\
& \leq 2 \epsilon^{-1}\left(\sqrt{b\left(x_{0}\right)}-\sqrt{b\left(y_{0}\right)}\right)^{2} \operatorname{trace}(C) \leq 2 \epsilon^{-1} \kappa_{b^{\frac{1}{2}}}^{2}\left|x_{0}-y_{0}\right|^{2} \operatorname{trace}(C) .
\end{aligned}
$$

A mere calculus gives that $\operatorname{trace}(C)=7\left|p_{0}\right|^{2}$, and finally,

$$
\operatorname{trace}\left(G\left(\begin{array}{cc}
X & 0 \\
0 & -Y
\end{array}\right)\right) \leq 14 \epsilon^{-1} \kappa_{b^{\frac{1}{2}}}^{2}\left|x_{0}-y_{0}\right|^{4} .
$$


Consequently, by applying Theorem 8.3 from [33] with the same notations as in the case $x_{0}=y_{0}$, we obtain that there exist $\tau_{1}, \tau_{2} \in \mathbb{R}$, and $X, Y \in \mathcal{S}^{2}$ such that

$$
\begin{aligned}
& \tau_{1}+c\left(x_{0},(\epsilon)^{-1}\left|p_{0}\right|^{2} p_{0}\right)+E\left(x_{0}, X\right)+F\left((\epsilon)^{-1}\left|p_{0}\right|^{2} p_{0}, X\right) \leq-\frac{\lambda}{T^{2}}, \\
& \tau_{2}+c\left(y_{0},(\epsilon)^{-1}\left|p_{0}\right|^{2} p_{0}\right)+E\left(y_{0}, Y\right)+F\left((\epsilon)^{-1}\left|p_{0}\right|^{2} p_{0}, Y\right) \geq 0 .
\end{aligned}
$$

So,

$$
\mid \begin{aligned}
\frac{\lambda}{T^{2}} & \leq c\left(y_{0},(\epsilon)^{-1}\left|p_{0}\right|^{2} p_{0}\right)-c\left(x_{0},(\epsilon)^{-1}\left|p_{0}\right|^{2} p_{0}\right)+E\left(y_{0}, Y\right)-E\left(x_{0}, X\right), \\
& \leq\left(\kappa_{d}+14 \kappa_{b^{\frac{1}{2}}}^{2}\right) \epsilon^{-1}\left|x_{0}-y_{0}\right|^{4}+\kappa_{h}\left|x_{0}-y_{0}\right|
\end{aligned}
$$

the function $c$ being locally Lipschitz in space. From this last inequality, we deduce that

$$
\lambda \leq\left(\kappa_{d}+14 \kappa_{b^{\frac{1}{2}}}^{2}\right) \epsilon^{-1} T^{2}\left|x_{0}-y_{0}\right|^{4}+\kappa_{h} T^{2}\left|x_{0}-y_{0}\right| .
$$

Denoting by $\vartheta=\max \left(\|u\|_{L^{\infty}\left(\mathbb{R}^{2}\right)},\|v\|_{L^{\infty}\left(\mathbb{R}^{2}\right)}\right)$, it can be easily proved that $\left|x_{0}-y_{0}\right| \leq$ $(8 \vartheta \epsilon)^{\frac{1}{4}}$.

Let us now denote by $M^{\prime}=\lim _{h \rightarrow 0} \sup _{|y-x| \leq h, t \in[0, T)}(u(x, t)-v(y, t))$ and $M_{h}=$ $\sup _{|y-x| \leq h}(u(x, t)-v(y, t))$. Let $\left(x_{n}^{h}, y_{n}^{h}, t_{n}^{h}\right)$ be such that $u\left(x_{n}^{h}, t_{n}^{h}\right)-v\left(y_{n}^{h}, t_{n}^{h}\right) \geq M_{h}-\frac{1}{n}$ with $\left|x_{n}^{h}-y_{n}^{h}\right| \leq h$. So,

$$
M_{h}-\frac{1}{n}-\frac{h^{4}}{4 \epsilon} \leq u\left(x_{n}^{h}, t_{n}^{h}\right)-v\left(y_{n}^{h}, t_{n}^{h}\right)-(4 \epsilon)^{-1}\left|x_{n}^{h}-y_{n}^{h}\right|^{4} \leq M_{0} \leq u\left(x_{0}, t_{0}\right)-v\left(y_{0}, t_{0}\right) .
$$

Letting $h$ tend to 0 , it yields

$$
M^{\prime}-\frac{1}{n} \leq u\left(x_{0}, t_{0}\right)-v\left(y_{0}, t_{0}\right) .
$$

Sending $\epsilon$ to 0 , it gives

$$
\begin{aligned}
M^{\prime}-\frac{1}{n} & \leq \lim _{\epsilon \rightarrow 0} \inf u\left(x_{0}, t_{0}\right)-v\left(y_{0}, t_{0}\right) \\
& \leq \lim _{\epsilon \rightarrow 0} \sup u\left(x_{0}, t_{0}\right)-v\left(y_{0}, t_{0}\right) \\
& \leq \lim _{\epsilon \rightarrow 0} \sup \sup _{|x-y| \leq(8 \vartheta \epsilon)^{\frac{1}{4}}, t \in[0, T)}(u(x, t)-v(y, t)), \\
& \leq \lim _{h \rightarrow 0} \sup \sup _{|x-y| \leq h, t \in[0, T)}(u(x, t)-v(y, t)) \\
& =M^{\prime}
\end{aligned}
$$

So $\lim _{\epsilon \rightarrow 0} u\left(x_{0}, t_{0}\right)-v\left(y_{0}, t_{0}\right)=M^{\prime}$. From $M^{\prime}-\frac{1}{n} \leq M_{0} \leq u\left(x_{0}, t_{0}\right)-v\left(y_{0}, t_{0}\right)$, we obtain $\lim _{\epsilon \rightarrow 0} M_{0}=M^{\prime}$.

Consequently, $\lim _{\epsilon \rightarrow 0}\left(u\left(x_{0}, t_{0}\right)-v\left(y_{0}, t_{0}\right)-M^{\prime}\right)=\lim _{\epsilon \rightarrow 0} \frac{\left|x_{0}-y_{0}\right|^{4}}{4 \epsilon}=0$.

Letting $\epsilon$ tend to 0 in (A.7), we obtain a contradiction. Therefore $M \leq 0$ and

$$
u \leq v \text { in } \mathbb{R}^{2} \times[0, T) .
$$




\section{REFERENCES}

[1] L. Alvarez, F.Guichard, P.L. Lions and J.M. Morel, Axioms and fundamental equations of image processing, Arch. Rational Mechan. Anal., 16 (IX), 200-257, 1993.

[2] L. Amodei and M.N. Benbourhim, A vector spline approximation, Journal of Approximation Theory, 67(1), 51-79, 1991.

[3] R. Arcangeli and M.C. López de Silanes, Multidimensional Minimizing Splines, Kluwer Academic Publishers, 2004.

[4] G. Aronsson, Minimization problems for the functional $\sup _{x} F\left(x, f(x), f^{\prime}(x)\right)$, Arkiv für Mate., 6, 33-53, 1965.

[5] G. Aronsson, Minimization problems for the functional $\sup _{x} F\left(x, f(x), f^{\prime}(x)\right)$. II, Arkiv für Mate., 6, 409-431, 1966.

[6] G. Aronsson, Extension of functions satisfying Lipschitz conditions, Arkiv für Mate., 6(6), 551-561, 1967.

[7] G. Aronsson, On the partial differential equation $u_{x}^{2} u_{x x}+2 u_{x} u_{y} u_{x y}+u_{y}^{2} u_{y y}=0$, Arkiv für Mate., 7, 395-425, 1968.

[8] G. Aronsson, Minimization problems for the functional $\sup _{x} F\left(x, f(x), f^{\prime}(x)\right)$. III, Arkiv für Mate., 7, 509-512, 1969.

[9] G. Aronsson, M. Crandall and P. Juutinen, A tour of the theory of absolutely minimizing functions, American Mathematical Society Bulletin. New Series, 41, 439-505, 2004.

[10] G. Aubert and P. Kornprobst, Mathematical Problems in Image Processing: Partial Differential Equations and the Calculus of Variations, Springer Verlag, 2002.

[11] J.F. Aujol and G. Aubert, Modeling very oscillating signals. Application to image processing, Applied Mathematics and Optimization, 51(2), 163-182, 2005.

[12] J.F. Aujol, G. Aubert, L. Blanc-Féraud and A. Chambolle, Image decomposition application to SAR images, Scale-Space , 297-312, 2003.

[13] J.F. Aujol, G. Aubert, L. Blanc-Féraud and A. Chambolle, Image decomposition into a bounded variation component and an oscillating component, Journal of Mathematical Imaging and Vision, 22, 71-88, 2005.

[14] G. Barles, Solutions de viscosité des équations de Hamilton-Jacobi, Springer Verlag, 1994.

[15] G. Barles, Solutions de viscosité et équations elliptiques du deuxième ordre, Cours de DEA, 1997.

[16] G. Barles and J. Busca, Existence and comparaison results for fully nonlinear degenerate elliptic equations without zeroth-order term, Comm. Partial Diff. Equations, 26, 2323-2337, 2001.

[17] E.N. Barron, L.C. Evans and R. Jensen, The infinity Laplacian, Aronsson's equation and their generalizations, Transactions of the American Mathematical Society, 360(1), 77-101, 2008.

[18] M.N. Benbourhim and A. Bouhamidi, Approximation of vector fields by thin plate splines with tension, Journal of Approximation Theory, 136, 198-229, 2005.

[19] M.N. Benbourhim and A. Bouhamidi, Div-free minimizing splines under tension, Prépublication, Institut de Mathématiques de Toulouse, 2007.

[20] M.N. Benbourhim and A. Bouhamidi, Error estimates for interpolating divcurl splines under tension on a bounded domain, Journal of Approximation Theory, 152, 66-81, 2008.

[21] H. Brézis, Analyse fonctionnelle, Dunod, 1999.

[22] J. Canny, A computational approach to edge detection, IEEE Pattern Anal. Machine Intell., PAMI-8, 679-698, 1986.

[23] E. Carlini, M. Falcone, N. Forcadel and R. Monneau, Convergence of a Generalized Fast Marching Method for an Eikonal equation with a Velocity Changing Sign, SIAM Journal on Numerical Analysis, 46(6), 2920-2952, 2008.

[24] S. Carlsson, Sketch based coding of grey level images, Signal Process, 15, 57-83, 1988.

[25] J.R. Casas, Image compression based on perceptual coding techniques, Ph.D. dissertation, Dept. Signal Theory Commun., UPC, Barcelona, Spain, 1996.

[26] J.R. Casas and L. Torres, Strong edge features for image coding, Mathematical Morphology and Its Applications to Image and Signal Processing, R.W. Schafer, P. Maragos, and M.A. Butt (Eds.), Boston, MA: Kluwer, 443-450, 1996.

[27] V. Caselles, F. Catté, C. Coll and F. Dibos, A geometric model for active contours in image processing, Numerische Mathematik., 66, 1-31, 1993.

[28] V. Caselles, R. Kimmel and G. Sapiro, Geodesic active contours, International Journal of Computer Vision, 22(1), 61-87, 1997.

[29] V. Caselles, J.M. Morel and C. Sbert, An axiomatic approach to image interpolation, IEEE Transactions on Image Processing, 7(3), 376-386, 1998.

[30] L.D. Cohen, On active contour models and balloons, Computer Vision, Graphics, and Image Processing: Image Understanding, 53(2), 211-218, 1989. 
[31] G. Cong, M. Esser, B. Parvin and G. Bebis, Shape metamorphism using p-Laplacian equation, ICPR, 4, 15-18, 2004.

[32] M.G. Crandall, A visit with the $\infty$-Laplace equation, Notes from a CIME course, preprint, 2005.

[33] M.G. Crandall, H. Ishii and P.L. Lions, User's guide to viscosity solutions of second order partial differential equations, Bulletin American Math. Soc., 27, 1-67, 1992.

[34] M.G. Crandall and P.L. Lions, Viscosity solutions of Hamilton-Jacobi Equations, Trans. Amer. Math. Soc., 277, 1-42, 1983.

[35] M.G. Crandall and P.L. Lions, On existence and uniqueness of solutions of Hamilton-Jacobi equations, Nonlinear Anal., 10, 353-370, 1986.

[36] F. Dodu and C. Rabut, Vectorial interpolation using radial-basis-like functions, Comput. Math. Appl., 43(3-5), 393-411, 2002.

[37] F. Dodu and C. Rabut, Irrotational or divergence free interpolation, Numer. math, 98, 477-498, 2004.

[38] C. Elion, and L.A. Vese, An image decomposition model using the total variation and the infinity Laplacian, Proceedings SPIE, 6498, 64980W-1-64980W-10, 2007.

[39] N. Forcadel, Contribution à l'analyse d'équations aux dérivées partielles décrivant le mouvement de fronts avec applications à la dynamique des dislocations, Ph.D., Ecole Nationale des Ponts et Chaussées, Marne La Vallée, France, 2007.

[40] N. Forcadel, Dislocations dynamics with a mean curvature term: short time existence and uniqueness, Differential and Integral Equations, 21(3-4), 285-304, 2008.

[41] N. Forcadel, C. Le Guyader and C. Gout, Generalized fast marching method: applications to image segmentation, Numerical Algorithms, 48, 189-211, 2008.

[42] L. Guillot and M. Bergounioux, Existence and uniqueness results for the Gradient Vector Flow and geodesic active contours mixed model, Comm. Pure Appl. Anal., to appear, 2008.

[43] W.S. Havens and J.C. Strikwerda, An improved operator for edge detection, 1984.

[44] H. Ishii, Existence and uniqueness of solutions of Hamilton-Jacobi equations, Funkcial. Ekvac., 29, 167-188, 1986

[45] H. Ishii and P.L. Lions, Viscosity solutions of fully nonlinear second-order elliptic partial differential equations, J. Differ. Equs., 83, 26-78, 1990.

[46] H. Ishii and M.-H. Sato, Nonlinear oblique derivative problems for singular degenerate parabolic equations on a general domain, Nonlinear Analysis, 57, 1077-1098, 2004.

[47] R. Jensen, P.L. Lions and P.E. Souganidis, A uniqueness result for viscosity solutions of second order fully nonlinear PDE, Proc. Amer. Math. Soc., 102(4), 975-978, 1988.

[48] R. Jensen, The maximum principle for viscosity solutions of fully nonlinear second order partial differential equations, Arch. Rational Mech. Analysis, 101(1), 1-27, 1988.

[49] R. Jensen, Uniqueness of Lipschitz extensions minimizing the sup-norm of the gradient, Arch. Rational Mech. Analysis, 123(1), 51-74, 1993.

[50] N. Jifeng, W. Chengke, L. Shigang and Y. Shuqin, NGVF: An improved external force field for active contour model, Pattern Recognition Letters, 28, 58-63, 2007.

[51] M. Kass, D. Terzopoulos and A. Witkin, Snakes: Active contour models, International Journal of Computer Vision, 1, 321-331, 1988.

[52] T.M. Le and L.A. Vese, Image decomposition using total variation and $\operatorname{div}(B M O)$, Multiscale Modeling and Simulation, 4(2), 390-423, 2005.

[53] C. Le Guyader, D. Apprato and C. Gout, On the construction of topology-preserving transformations, SIAM conference on Imaging Science, San Diego, 2008.

[54] F. Mémoli, G. Sapiro and P. Thompson, Brain and surface warping via minimizing Lipschitz extensions, MFCA, International Workshop on Mathematical Foundations of Computational Anatomy, 2006.

[55] Y. Meyer, Oscillating Patterns in Image Processing and Nonlinear Evolution Equations, AMS, $22,2001$.

[56] S. Osher and R. Fedkiw, Level Set Methods and Dynamic Implicit Surfaces, Springer Verlag, 2003.

[57] S. Osher and J.A. Sethian, Fronts propagation with curvature dependent speed: Algorithms based on Hamilton-Jacobi formulations, J. Comput. Phys., 79, 12-49, 1988.

[58] N. Paragios, O. Mellina-Gottardo and V. Ramesh, Gradient Vector Flow Fast Geodesic Active Contours, Proc. IEEE Intl Conf. Computer Vision, 1, 67-73, 2001.

[59] J.M.S. Prewitt, Object enhancement and extraction, Picture Processing and Psychopictorics, B. Lipkin and A. Rosenfeld (eds.), New York: Academic, 75-149, 1970.

[60] J.A. Sethian, Level Set Methods and Fast Marching Methods: Evolving interfaces in Computational Geometry, Fluid Mechanics, Computer Vision and Material Science, Cambridge University Press, Londres, 1999. 
[61] V. Torre and T.A. Poggio, On edge detection, IEEE Trans. Pattern Anal. Machine Intell., PAMI-8, 147-163, 1986.

[62] L.A. Vese and S.J. Osher, Modeling textures with total variation minimization and oscillating patterns in image processing, J. Sci. Comput., 19(1-3), 553-572, 2003.

[63] L.A. Vese and S.J. Osher, Image denoising and decomposition with total variation minimization and oscillatory functions, Journal of Mathematical Imaging and Vision, 20(1-2), 7-18, 2004.

[64] C. Xu and J.L. Prince, Gradient vector flow: a new external force model for snakes, IEEE Proc. Conf. on Computer Vision and Pattern Recognition, 66-71, 1997.

[65] C. Xu and J.L. Prince, Snakes, shapes, and gradient vector flow, IEEE Trans. Image Process., 7(3), 359-369, 1998.

[66] A.L. Yuille and T.A. Poggio, Scaling theorems for zero-crossings, IEEE Trans. Pattern Anal. Machine Intell., PAMI-8, 15-25, 1986. 